\title{
Sulphur-bearing species as chemical clocks for low mass protostars?
}

\author{
J. V. Buckle ${ }^{1,2}$ and G. A. Fuller ${ }^{1}$ \\ 1 Department of Physics, UMIST, PO Box 88, Manchester M60 1QD, UK \\ 2 Joint Astronomy Centre, 660 North A'ohoku Place University Park Hilo, HI 96720, USA \\ e-mail: G.Fuller@umist.ac.uk
}

Received 16 October 2001 / Accepted 5 December 2002

\begin{abstract}
We present observations of $\mathrm{H}_{2} \mathrm{~S}, \mathrm{SO}$ and $\mathrm{SO}_{2}$ towards a sample of Class 0 and Class I sources. The $\mathrm{H}_{2} \mathrm{~S}$ abundances rule out its formation in the gas phase and we assume that it is released from grain mantles. Applying a model of the evolution of the sulphur chemistry first used in the study of hot cores, we show that this model can reproduce the results of our observations even for the relatively low temperatures and densities of these sources. We demonstrate that the chemical evolution of sulphur bearing species is a potentially valuable probe of chemical timescales in low mass star forming regions. Overall, the model indicates that Class I sources are at a later chemical evolutionary stage than their Class 0 counterparts, with an average difference in chemical age of at least $3 \times 10^{3}$ years and an upper limit on the age of the Class I sources of a few times $10^{5}$ years.
\end{abstract}

Key words. stars: formation - radio lines: ISM - ISM: abundances - astrochemistry

\section{Introduction}

Young stars have a significant impact on the chemistry in the dense molecular clouds in which they form. This is particularly true for hot cores, where the high temperatures and densities act to drive a rapid chemistry, subsequent to the heating of the core during collapse (Hatchell et al. 1998; Charnley 1997). The raised temperatures release molecules from the icy mantles on grains, and so provide fuel for chemical networks. Despite the lower temperatures and densities, observations have shown that the regions surrounding low mass protostars also have a rich chemistry (van Dishoeck \& Hogerheijde 1999). The same processes of energetic outflow interactions forming shocked regions, and increased densities and temperatures evaporating ice mantles from grains, have been observed towards these low mass sources. Since much of the chemistry is driven by the changing conditions during star formation, it should be possible to place constraints on chemical timescales within low mass protostellar regions.

The chemical networks driven by the evaporation of molecules may provide a method of assessing protostellar ages through the chemical evolution of the envelope. Chemical reactions proceed, driven by the molecules evaporated from ice mantles on dust grains, and abundances change as the released molecules form other species. Comparison of the observed abundances with those predicted by chemical models, can

Send offprint requests to: J. V. Buckle, e-mail: j.buckle@jach.hawaii.edu provide constraints on the time since the ice mantles first evaporated. The use of molecular abundances as chemical clocks has been investigated in hot core regions (Hatchell et al. 1998), where the high temperatures and densities move the chemistry forward rapidly. In particular, sulphur-bearing species provide a rapidly evolving chemistry, with a chemical reaction network that has been studied in detail.

To investigate whether sulphur species might provide a chemical clock for low mass stars we have undertaken a survey, described and analysed below, of three sulphur containing species towards low mass Class 0 and I sources.

\section{Observations and data reduction}

We have observed a sample of 10 Class 0 and 9 Class I sources in three sulphur bearing molecules, $\mathrm{H}_{2} \mathrm{~S}, \mathrm{SO}$ and $\mathrm{SO}_{2}$. The observations were carried out at the National Radio Astronomy Observatory (NRAO) ${ }^{1} 12 \mathrm{~m}$ telescope on Kitt Peak, Arizona, in June 2000, using the $2 \mathrm{~mm}$ and $3 \mathrm{~mm}$ SIS receivers and the millimetre auto-correlator (MAC) spectrometer in 4 IF mode with 8000 channels. The spectra were observed using $15 \mathrm{kHz}$ channels, giving a velocity resolution $\sim 0.03 \mathrm{~km} \mathrm{~s}^{-1}$. The observations were made in two polarization channels and these were averaged. In order to constrain the molecular abundances, we also observed $\mathrm{C}^{18} \mathrm{O}$ using the $3 \mathrm{~mm}$ receiver, and the MAC

\footnotetext{
1 The National Radio Astronomy Observatory is a facility of the National Science Foundation operated under cooperative agreement by Associated Universities, Inc.
} 
Table 1. Molecular data for observed molecules.

\begin{tabular}{llccccc}
\hline \hline Molecule & Transition & $\begin{array}{c}\text { Frequency } \\
\mathrm{MHz}\end{array}$ & $\begin{array}{c}E_{\text {lower }} \\
\mathrm{K}\end{array}$ & $\begin{array}{c}\mu \\
\text { Debye }\end{array}$ & $S$ & $\eta_{\mathrm{m}}^{*}$ \\
\hline $\mathrm{H}_{2} \mathrm{~S}$ & $J_{k-, k+}=1_{1,0} \rightarrow 1_{0,1}$ & 168762.76 & 19.78 & 0.97 & 4.50 & 0.76 \\
$\mathrm{SO}$ & $J_{N}=2_{2} \rightarrow 1_{1}$ & 86093.95 & 15.81 & 1.55 & 3.94 & 0.89 \\
$\mathrm{SO}$ & $J_{N}=4_{3} \rightarrow 3_{2}$ & 138178.60 & 9.23 & 1.55 & 3.38 & 0.76 \\
$\mathrm{SO}_{2}$ & $J_{k-, k_{+}}=5_{1,5} \rightarrow 4_{0,5}$ & 135696.02 & 9.15 & 1.63 & 3.13 & 0.76 \\
${ }^{34} \mathrm{SO}$ & $J_{N}=4_{3} \rightarrow 3_{2}$ & 135775.31 & 9.09 & 1.55 & 3.94 & 0.76 \\
${ }^{34} \mathrm{SO}$ & $J_{N}=4_{4} \rightarrow 3_{3}$ & 168815.51 & 25.30 & 1.55 & 3.75 & 0.76 \\
$\mathrm{C}^{18} \mathrm{O}$ & $J=1 \rightarrow 0$ & 109782.17 & 0.00 & 0.11 & 1.00 & 0.97 \\
\hline
\end{tabular}

in 2IF mode with 16000 channels. The data were taken in position switching mode, with offset positions of $\pm 15^{\prime}$. The effective system temperatures ranged between 300 to $500 \mathrm{~K}$ during the observations. The molecular transitions observed, with molecular data obtained from the JPL molecular line database (Pickett et al. 1998), are listed in Table 1, along with the corrected main beam efficiencies, $\eta_{\mathrm{m}}^{*}$, of the telescope.

The sources, listed in Table 2, all have enhanced abundances of $\mathrm{CH}_{3} \mathrm{OH}$, suggesting that grain mantle evaporation plays an important role in the chemical networks for these sources (Buckle \& Fuller 2002). The sources have a range of bolometric temperatures, allowing us to evaluate the ability of the sulphur chemical network to act as an evolutionary probe of low mass protostars. In addition to observations at the source position (referred to as core positions), we have made a detailed study of the regions adjacent to to S68N in the Serpens Molecular Cloud. In this region, we have observed at the peak of several high velocity CO $J=2 \rightarrow 1$ clumps (Davis et al. 1999), which are listed in Table 2 as arc-second offsets from S68N, and are referred to as outflow positions. The positions observed towards Serpens have been marked in Fig. 1, with the approximate $F W H M$ size of the NRAO beam at $168 \mathrm{GHz}, 43^{\prime \prime}$, indicated.

Sample spectra from each of the molecules towards the sources observed are given in Fig. 2. The complete set of spectra towards these sources are shown in Buckle (2001).

Data reduction was carried out using the radio data reduction software CLASS. One or two Gaussian components were fitted to the data using the CLASS routines. For a few sources, visual inspection of the line profiles indicated that two Gaussian components were necessary to provide the best fit to the line. For other sources, the decision to make a one or two component fit was made on the basis of the residuals after removing the fit from the spectrum. If a double component line that produced the best fit was the sum of two similar linewidth and velocity components, a single component fit was used.

\section{Data analysis}

$\mathrm{H}_{2} \mathrm{~S}$ was detected towards all of the observed positions, with peak intensities $T_{\mathrm{R}}^{*}<1 \mathrm{~K}$. The core positions have the strongest emission, with a similar range of peak intensities between Class 0 and Class I sources. Towards the outflow positions in Serpens, the emission tends to be weaker than towards the core positions.
Table 2. Sources observed, with bolometric temperatures, distances to the source and positions. For sources where the bolometric temperature is unknown, the source has been labelled as Class 0 or Class I in parentheses.

\begin{tabular}{lrrrr}
\hline \hline Source & $\begin{array}{r}D \\
(\mathrm{pc})\end{array}$ & $\begin{array}{r}T_{\text {bol }} \\
(\mathrm{K})\end{array}$ & $\begin{array}{r}\text { RA } \\
(1950)\end{array}$ & $\begin{array}{r}\text { DEC } \\
(1950)\end{array}$ \\
\hline IRAS 03282+3035 & 300 & 26 & $03: 28: 15.2$ & $30: 35: 14$ \\
IRAS 04166+2706 & 140 & 139 & $04: 16: 37.8$ & $27: 06: 29$ \\
IRAS 04169+2702 & 140 & 170 & $04: 16: 53.8$ & $27: 02: 52$ \\
IRAM 04191+1522 & 140 & 18 & $04: 19: 06.4$ & $15: 22: 45$ \\
L1551 IRS5 & 140 & 97 & $04: 28: 40.2$ & $18: 01: 42$ \\
L1551 NE & 140 & 75 & $04: 28: 50.8$ & $18: 02: 11$ \\
L1535 & 140 & 152 & $04: 32: 33.4$ & $24: 02: 13$ \\
TMR-1 & 140 & 144 & $04: 36: 09.7$ & $25: 47: 29$ \\
L1527 & 140 & 59 & $04: 36: 49.3$ & $25: 57: 16$ \\
IRAS 04381+2540 & 140 & 139 & $04: 38: 08.5$ & $25: 40: 53$ \\
S68N & 300 & 40 & $18: 27: 15.6$ & $01: 14: 42$ \\
blue lobe peak & & & $339^{\prime \prime}$ & $146^{\prime \prime}$ \\
red lobe peak & & & $366^{\prime \prime}$ & $146^{\prime \prime}$ \\
red lobe peak & & & $278^{\prime \prime}$ & $-7^{\prime \prime}$ \\
blue lobe peak & & & $143^{\prime \prime}$ & $190^{\prime \prime}$ \\
blue lobe peak & & & $-138^{\prime \prime}$ & $89^{\prime \prime}$ \\
SMM1 & 300 & 51 & $18: 27: 17.5$ & $01: 12: 13$ \\
SMM4 & 300 & 43 & $18: 27: 24.8$ & $01: 11: 08$ \\
SMM11 & 300 & $(0)$ & $18: 27: 27.9$ & $01: 09: 37$ \\
SMM8 & 300 & $(0)$ & $18: 27: 34.2$ & $01: 14: 30$ \\
B335 & 250 & 37 & $19: 34: 35.7$ & $07: 27: 20$ \\
L1152 & 440 & 72 & $20: 35: 19.4$ & $67: 42: 30$ \\
L1157 & 440 & 62 & $20: 38: 39.6$ & $67: 51: 33$ \\
L1262 & 200 & 104 & $23: 23: 48.7$ & $74: 01: 08$ \\
\hline & & & & \\
& & & & \\
& & & & \\
& & & &
\end{tabular}

The SO $J_{N}=4_{3} \rightarrow 3_{2}$ transition, which has lower state $\left(E_{1}\right)$ $9 \mathrm{~K}$ above ground, was detected towards $91 \%$ of the observed positions. Emission from this molecule is strong towards all of the sources where it was detected, with peak intensities of $1-2 \mathrm{~K}$. The $J_{N}=2_{2} \rightarrow 1_{1}$ transition, which has a lower state $15 \mathrm{~K}$ above ground, was detected towards only $25 \%$ of the observed positions, with peak intensities $<0.3 \mathrm{~K}$. Emission from ${ }^{34} \mathrm{SO}$ was very weak $\left(T_{\mathrm{R}}^{*}<0.4 \mathrm{~K}\right)$, with the $J_{N}=4_{3} \rightarrow 3_{2}$ transition $\left(E_{1}=9 \mathrm{~K}\right)$ detected towards only $39 \%$ of the observed positions, indicating that, despite the strength of the main isotopic SO line, in most sources this molecule is at most 

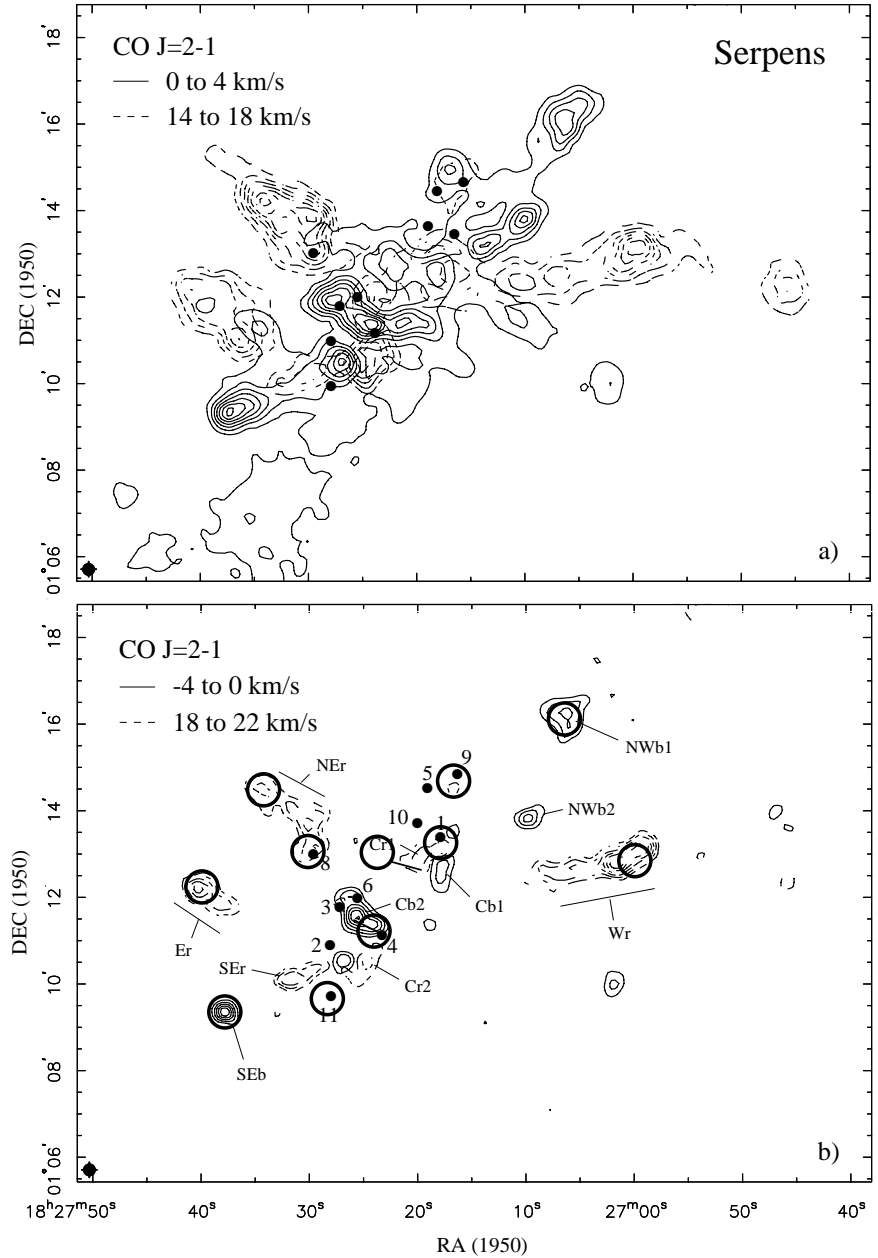

Fig. 1. Positions observed in Serpens (reproduced from Davis et al. 1999). Top: low velocity CO $J=2 \rightarrow 1$ emission. Bottom: high velocity CO $J=2 \rightarrow 1$ emission, overlaid with the positions observed in sulphur-bearing species. The open circles denote the approximate size of the NRAO beam at $168 \mathrm{GHz}$.

moderately optically thick. The ${ }^{34} \mathrm{SO} J_{N}=4_{4} \rightarrow 3_{3}$ transition $\left(E_{1}=25 \mathrm{~K}\right)$ was not detected towards any source.

$\mathrm{SO}_{2}$ was detected towards $30 \%$ of the observed positions, with weak emission from most of the sources. Peak intensities range from $0.1-0.4 \mathrm{~K}$. The sources with the highest peak intensities are two of the Serpens core positions, which also have strong emission from $\mathrm{H}_{2} \mathrm{~S}$ and $\mathrm{SO}$.

From the Gaussian fits to the data, linewidths, velocities, intensities and associated uncertainties are listed in Table 3. The integrated intensity was calculated using :

$\int T_{\mathrm{R}}^{*} \mathrm{~d} v=1.06 T_{\mathrm{R}}^{*} \Delta v$

where $\Delta v$ is the line $F W H M$ width, and $T_{\mathrm{R}}^{*}$ is the peak intensity. In addition, based on non-detections of molecular lines, we have also calculated upper limits to the integrated intensity, based on the noise in the spectrum, and assuming a linewidth of $1 \mathrm{~km} \mathrm{~s}^{-1}$. These values, for the core positions, are listed in Table 4.

\subsection{Linewidth and velocity components}

In order to compare the chemical evolution of molecular material surrounding the sources, we need to be clear that the molecular lines detected towards each position are tracing material in closely associated regions. Although $\mathrm{H}_{2} \mathrm{~S}$ and $\mathrm{SO}, \mathrm{SO}_{2}$ may not be tracing exactly the same gas, due to the differences in the expected chemical formation routes (Sect. 3.4), we would not expect to see large differences in, for example, the linewidths and velocities of molecular lines observed towards each position. Figure 3 illustrates that, towards many of the sources we observed, the similar linewidths and velocities indicate that these transitions are tracing emission arising in the same kinematic regions.

However, there are discrepancies towards some of the sources. For example, S68N mainly has line components at $\sim 8.5 \mathrm{~km} \mathrm{~s}^{-1}$, although emission from SO $J_{k}=2_{2} \rightarrow 1_{1}$ has an extended second component to the red of the line peak, and emission from $\mathrm{C}^{18} \mathrm{O}$ has a small wing redward of the line peak. The regions where emission from the second SO and $\mathrm{C}^{18} \mathrm{O}$ components arise, characterised by different central velocities and linewidths, is unlikely to be closely associated with emission traced by the rest of the molecular transitions with $1 \mathrm{~km} \mathrm{~s}^{-1}<\Delta v<5 \mathrm{~km} \mathrm{~s}^{-1}$ and $8.3 \mathrm{~km} \mathrm{~s}^{-1}<v_{0}<$ $9.0 \mathrm{~km} \mathrm{~s}^{-1}$. Therefore, in calculating the fractional abundances towards S68N, we use the components marked by $*$ in Table 3. Similarly, emission from $\mathrm{C}^{18} \mathrm{O}$ towards IRAS $03282+3035$ has a wing blueward of the line peak, which we fit as a second component. This second component in $\mathrm{C}^{18} \mathrm{O}$ has no counterpart in any of the other molecular lines we detected towards IRAS $03282+3035$, and so we do not use data derived from this component of the fit in calculating the molecular abundances. For L1527, we have summed the integrated intensity of emission from $\mathrm{H}_{2} \mathrm{~S}$ rather than split it into components, as we do not see similar components in any other molecule towards this source.

We calculate fractional abundances for comparison with the model only from those regions where the linewidth and velocity comparison suggests the molecular emission is tracing the same region. Of the two, we have weighted the line centre velocity more heavily than the linewidth. The components used to calculate fractional abundances towards the sources we observed have been marked with matching symbols $(*$ or $* *)$ in Table 3.

\subsection{Temperatures and column densities}

The column densities are calculated assuming that the molecules are in local thermodynamic equilibrium (LTE) and fill the telescope beam. Assuming also that the emission is optically thin,

$\frac{3 k \int T_{\mathrm{mb}} \mathrm{d} v}{8 \pi^{3} \mu^{2} v S}=\frac{N_{\mathrm{mol}}}{Q\left(T_{\mathrm{ex}}\right)} \mathrm{e}^{-E_{\mathrm{u}} / k T_{\mathrm{ex}}}$

$N_{\mathrm{mol}}=\frac{3 k \int T_{\mathrm{mb}} \mathrm{d} v Q\left(\frac{T_{\mathrm{rot}}}{3 \mathrm{k}}\right) \mathrm{e}^{E_{\mathrm{u}} / T_{\mathrm{ex}}}}{8 \pi^{3} \mu^{2} v S}$

where $T_{\mathrm{mb}}$ is calculated from the corrected source antenna temperature, $T_{\mathrm{R}}^{*}$, using the corrected main beam efficiency, 

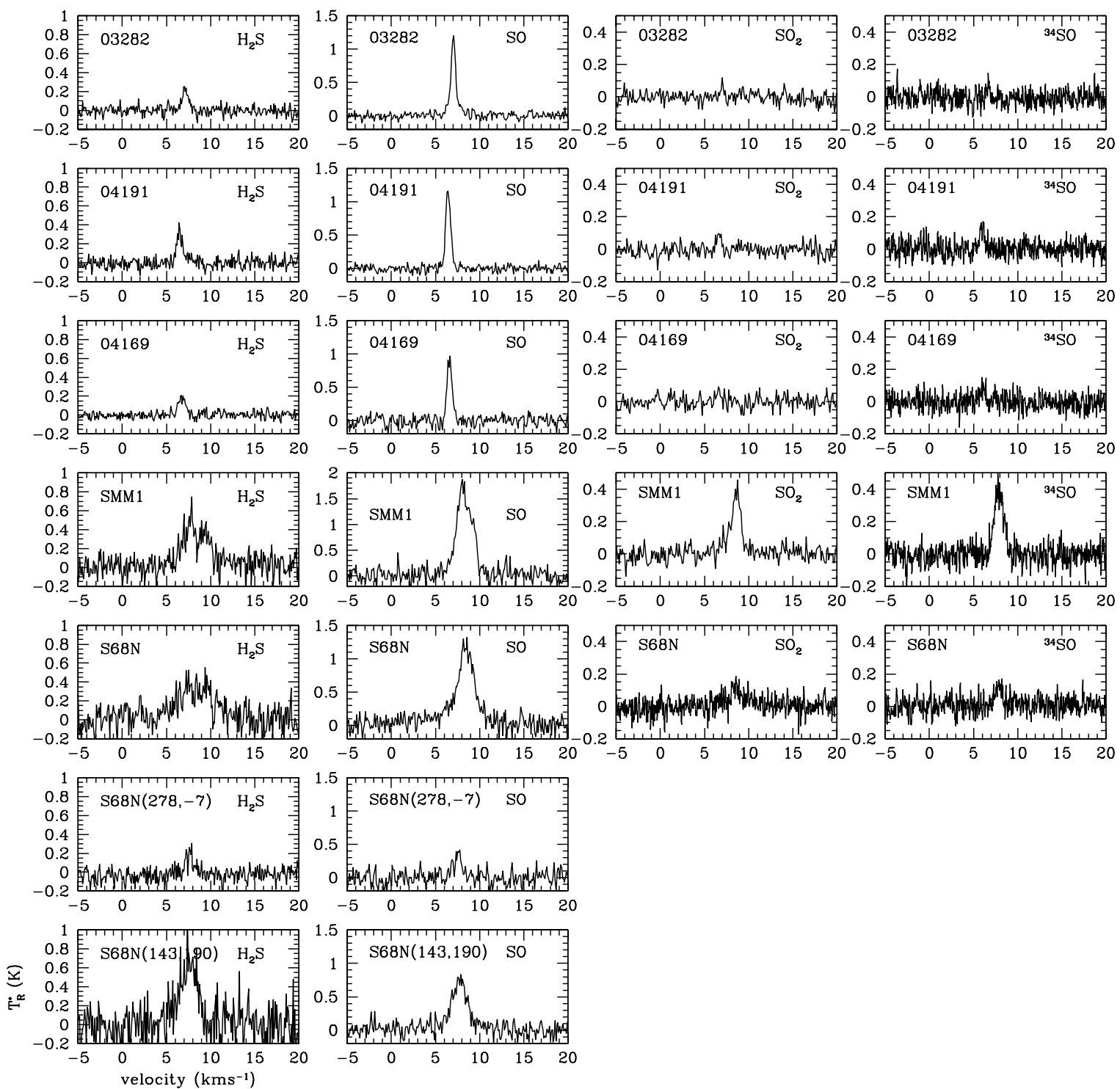

Fig. 2. Sample spectra of $\mathrm{H}_{2} \mathrm{~S}$, SO $J_{N}=4_{3} \rightarrow 3_{2}, \mathrm{SO}_{2}$, and ${ }^{34} \mathrm{SO} J_{k}=4_{3} \rightarrow 3_{2}$.

$T_{\mathrm{mb}}=T_{\mathrm{R}}^{*} / \eta_{\mathrm{m}}^{*} \cdot \int T_{\mathrm{mb}} \mathrm{d} v$ is the integrated line intensity, $\mu$ is the transition dipole moment, $S$ is the rotational line strength, $N_{\text {mol }}$ is the total molecular column density, and $E_{\mathrm{u}}$ is the upper energy level. All the molecular constants were obtained from the JPL molecular line database (Pickett et al. 1998). The excitation temperature, $T_{\mathrm{ex}}$, has been taken to be $10 \mathrm{~K}$, a value appropriate to these low mass protostars (Ladd et al. 1998; van Dishoeck \& Blake 1998).

An alternative calculation of the column density approximates the rotation temperature as $\frac{2 E_{u}}{3 k}$ for non-linear molecules, and $\frac{E_{\mathrm{u}}}{k}$ for linear molecules to calculate the minimum column density (Thompson et al. 1999). The column densities calculated for $10 \mathrm{~K}$ are always within a factor of 2 or less of these minimum column densities.

The $\mathrm{C}^{18} \mathrm{O}$ column densities vary from a few $\times 10^{14} \mathrm{~cm}^{-2}$ to a few $\times 10^{15} \mathrm{~cm}^{-2}$. These have been used to determine the total $\mathrm{H}_{2}$ column densities, in $\mathrm{cm}^{-2}$, using (Frerking et al. 1982):

$N\left(\mathrm{H}_{2}\right)=5.53 \times 10^{6} N\left(\mathrm{C}^{18} \mathrm{O}\right)+1.22 \times 10^{21}$ giving $\mathrm{H}_{2}$ column densities of a few $\times 10^{21} \mathrm{~cm}^{-2}$ to a few $\times 10^{22} \mathrm{~cm}^{-2}$.

Table 3 displays the derived column densities for the sources listed in Table $2 . \mathrm{H}_{2} \mathrm{~S}$, due to the two hydrogen spins, possesses both ortho and para species. The detected transition is an ortho species, and so the column densities have been calculated for the ortho levels only. To estimate the total column density, assuming an ortho to para ratio of 3:1 (Turner 1996; Tieftrunk et al. 1994), the ortho column density has been multiplied by $4 / 3$, and then listed in Table 3 .

We can also estimate the ${ }^{32} \mathrm{SO}$ column densities from our observations of ${ }^{34} \mathrm{SO}$, assuming the latter is optically thin, and rarer by a factor of 20 than ${ }^{32} \mathrm{SO}$ (Hatchell et al. 1998). We did not detect emission from ${ }^{34} \mathrm{SO}$ towards the majority of sources, indicating that ${ }^{32} \mathrm{SO}$ emission is optically thin towards most sources. Where we did detect emission from ${ }^{34} \mathrm{SO}$, the ${ }^{32} \mathrm{SO}$ column densities estimated from the ${ }^{34} \mathrm{SO}$ column densities are mainly between 1 and 3 times the column densities derived directly from ${ }^{32} \mathrm{SO}$ emission, indicating that emission 
Table 3. Velocities, intensities, rotation temperature approximations, minimum column densities and abundances for the molecular line observations, from Gaussian fits to the data. Uncertainties are quoted in parentheses. Offsets are in arc-seconds. For sources with two component lines, the narrower component is listed immediately below the broader component. For each source with more than one component, column densities which have been used to derive fractional abundances have been marked with matching symbols $(*$ or $* *)$.

\begin{tabular}{|c|c|c|c|c|c|c|c|}
\hline Source & Molecule & & $\begin{array}{r}\Delta v \\
\mathrm{~km} \mathrm{~s}^{-1}\end{array}$ & $\begin{array}{r}v \\
\mathrm{~km} \mathrm{~s}^{-1}\end{array}$ & $\begin{array}{r}\int T_{\mathrm{R}}^{*} \mathrm{~d} v \\
\mathrm{~K} \mathrm{~km} \mathrm{~s}{ }^{-1}\end{array}$ & $\begin{array}{r}N_{\mathrm{mol}} \\
\times 10^{13} \mathrm{~cm}^{-2}\end{array}$ & $\begin{array}{r}X_{\mathrm{mol}} \\
\times 10^{-9}\end{array}$ \\
\hline \multirow[t]{8}{*}{ I03282(0,0) } & $\mathrm{H}_{2} \mathrm{~S}_{1,0} \rightarrow 1_{0,1}$ & $*$ & $0.84(.06)$ & $7.12(.02)$ & $0.20(.01)$ & 0.44 & 0.77 \\
\hline & $\mathrm{SO} 4_{3} \rightarrow 3_{2}$ & * & $1.48(.14)$ & $7.06(.04)$ & $0.47(.04)$ & 0.67 & 1.19 \\
\hline & & $*$ & $0.47(.02)$ & $7.02(.01)$ & $0.45(.04)$ & 0.64 & 1.14 \\
\hline & $\mathrm{SO} 2_{2} \rightarrow 1_{1}$ & * & $0.38(.06)$ & $7.12(.03)$ & $0.09(.01)$ & 0.65 & 1.16 \\
\hline & $\mathrm{SO}_{2} 5_{1,4} \rightarrow 4_{0,4}$ & $*$ & $0.17(.04)$ & $7.03(.02)$ & $0.02(.00)$ & 0.07 & 0.12 \\
\hline & ${ }^{34} \mathrm{SO}_{4} \rightarrow 3_{2}$ & $*$ & $0.39(.09)$ & $6.67(.04)$ & $0.03(.01)$ & 0.04 & 0.08 \\
\hline & $\mathrm{C}^{18} \mathrm{O} 1 \rightarrow 0$ & $*$ & $0.95(.03)$ & $7.48(.01)$ & $0.89(.02)$ & 80.34 & \\
\hline & & & $0.51(.19)$ & $8.48(.06)$ & $0.05(.02)$ & 4.51 & \\
\hline \multirow[t]{4}{*}{ I04166 } & $\mathrm{H}_{2} \mathrm{~S} 1_{1,0} \rightarrow 1_{0,1}$ & $*$ & $0.88(.14)$ & $6.69(.04)$ & $0.09(.01)$ & 0.20 & 0.44 \\
\hline & $\mathrm{SO} 4_{3} \rightarrow 3_{2}$ & * & $0.43(.02)$ & $6.63(.01)$ & $0.15(.01)$ & 0.21 & 0.48 \\
\hline & $\mathrm{C}^{18} \mathrm{O} 1 \rightarrow 0$ & $*$ & $0.55(.01)$ & $6.84(.01)$ & $0.65(.01)$ & 58.67 & \\
\hline & & & $0.49(.04)$ & $5.56(.01)$ & $0.24(.01)$ & 21.66 & \\
\hline \multirow[t]{7}{*}{ I04169 } & $\mathrm{H}_{2} \mathrm{~S}_{1,0} \rightarrow 1_{0,1}$ & $*$ & $0.97(.05)$ & $6.75(.02)$ & $0.20(.01)$ & 0.44 & 40.94 \\
\hline & $\mathrm{SO} 4_{3} \rightarrow 3_{2}$ & $*$ & $0.66(.02)$ & $6.60(.01)$ & $0.66(.02)$ & 0.96 & 2.05 \\
\hline & $\mathrm{SO} 2_{2} \rightarrow 1_{1}$ & $*$ & $0.54(.07)$ & $6.75(.04)$ & $0.09(.01)$ & 0.60 & 1.42 \\
\hline & $\mathrm{SO}_{2} 5_{1,4} \rightarrow 4_{0,4}$ & $*$ & $0.44(.15)$ & $6.61(.05)$ & $0.04(.01)$ & 0.14 & 0.30 \\
\hline & ${ }^{34} \mathrm{SO}_{4} \rightarrow 3_{2}$ & $*$ & $0.75(.11)$ & $5.99(.06)$ & $0.06(.01)$ & 0.09 & 0.19 \\
\hline & $\mathrm{C}^{18} \mathrm{O} 1 \rightarrow 0$ & & $1.73(.18)$ & $6.89(.07)$ & $0.52(.07)$ & 46.94 & \\
\hline & & * & $0.59(.03)$ & $6.72(.01)$ & $0.68(.06)$ & 61.38 & \\
\hline \multirow[t]{6}{*}{ I04381 } & $\mathrm{H}_{2} \mathrm{~S} 1_{1,0} \rightarrow 1_{0,1}$ & $* *$ & $0.64(.10)$ & $6.10(.05)$ & $0.05(.01)$ & 0.11 & 0.42 \\
\hline & & $*$ & $0.41(.05)$ & $5.23(.02)$ & $0.05(.01)$ & 0.11 & 0.15 \\
\hline & $\mathrm{SO} 4_{3} \rightarrow 3_{2}$ & $*$ & $0.48(.06)$ & $5.18(.02)$ & $0.14(.01)$ & 0.20 & 0.28 \\
\hline & & $*$ & $0.33(.06)$ & $5.83(.03)$ & $0.06(.01)$ & 0.09 & 0.12 \\
\hline & $\mathrm{C}^{18} \mathrm{O} 1 \rightarrow 0$ & $*$ & $0.45(.01)$ & $5.37(.00)$ & $1.21(.02)$ & 109.22 & \\
\hline & & $* *$ & $0.34(.02)$ & $6.17(.01)$ & $0.27(.01)$ & 24.37 & \\
\hline \multirow[t]{6}{*}{ IRAM 04191(0,0) } & $\mathrm{H}_{2} \mathrm{~S}_{1,0} \rightarrow 1_{0,1}$ & $*$ & $0.77(.05)$ & $6.48(.02)$ & $0.27(.01)$ & 0.59 & 3.23 \\
\hline & $\mathrm{SO} 4_{3} \rightarrow 3_{2}$ & * & $0.66(.01)$ & $6.44(.00)$ & $0.81(.01)$ & 1.16 & 6.37 \\
\hline & $\mathrm{SO}_{2} 5_{1,4} \rightarrow 4_{0,4}$ & $*$ & $0.58(.08)$ & $6.63(.04)$ & $0.06(.01)$ & 0.58 & 1.13 \\
\hline & ${ }^{34} \mathrm{SO}_{3} \rightarrow 3_{2}$ & * & $0.62(.07)$ & $5.97(.03)$ & $0.09(.01)$ & 0.13 & 0.71 \\
\hline & $\mathrm{C}^{18} \mathrm{O} 1 \rightarrow 0$ & & $0.72(.02)$ & $6.71(.02)$ & $1.15(.06)$ & 103.81 & \\
\hline & & * & $0.33(.07)$ & $6.49(.03)$ & $0.12(.06)$ & 10.83 & \\
\hline \multirow[t]{5}{*}{ L1551IRS5 } & $\mathrm{H}_{2} \mathrm{~S}_{1,0} \rightarrow 1_{0,1}$ & $*$ & $1.05(.05)$ & $6.48(.02)$ & $0.34(.01)$ & 0.74 & 1.45 \\
\hline & $\mathrm{SO} 4_{3} \rightarrow 3_{2}$ & $*$ & $2.08(.61)$ & $6.47(.25)$ & $0.16(.05)$ & 0.23 & 0.45 \\
\hline & & * & $0.76(.14)$ & $6.42(.03)$ & $0.16(.05)$ & 0.23 & 0.45 \\
\hline & $\mathrm{C}^{18} \mathrm{O} 1 \rightarrow 0$ & & $1.07(.11)$ & $6.01(.12)$ & $0.35(.09)$ & 31.59 & \\
\hline & & $*$ & $0.61(.03)$ & $6.48(.01)$ & $0.78(.09)$ & 70.41 & \\
\hline \multirow[t]{5}{*}{$\operatorname{L} 1551 N E(0,0)$} & $\mathrm{H}_{2} \mathrm{~S} 1_{1,0} \rightarrow 1_{0,1}$ & $*$ & $1.96(.10)$ & $7.22(.05)$ & $0.33(.02)$ & 0.72 & 1.59 \\
\hline & $\mathrm{SO} 4_{3} \rightarrow 3_{2}$ & $*$ & $2.40(.22)$ & $6.93(.12)$ & $0.27(.03)$ & 0.39 & 0.86 \\
\hline & & $* *$ & $0.44(.06)$ & $6.54(.02)$ & $0.10(.01)$ & 0.14 & 0.27 \\
\hline & $\mathrm{C}^{18} \mathrm{O} 1 \rightarrow 0$ & $* *$ & $0.67(.04)$ & $6.63(.02)$ & $0.82(.02)$ & 74.02 & \\
\hline & & $*$ & $0.43(.01)$ & $6.78(.01)$ & $0.66(.02)$ & 56.58 & \\
\hline \multirow[t]{3}{*}{$\mathrm{L} 1535(0,0)$} & $\mathrm{H}_{2} \mathrm{~S}_{1,0} \rightarrow 1_{0,1}$ & * & $0.87(.10)$ & $5.84(.04)$ & $0.13(.01)$ & 0.28 & 0.44 \\
\hline & $\mathrm{SO} 4_{3} \rightarrow 3_{2}$ & $*$ & $0.54(.06)$ & $5.71(.04)$ & $0.10(.01)$ & 0.14 & 0.22 \\
\hline & $\mathrm{C}^{18} \mathrm{O} 1 \rightarrow 0$ & $*$ & $0.72(.02)$ & $5.82(.01)$ & $1.03(.03)$ & 92.98 & \\
\hline
\end{tabular}

from ${ }^{32} \mathrm{SO}$ is, at most, moderately optically thick towards these sources.

For those sources observed in $\mathrm{C}^{18} \mathrm{O}$ Table 3 also gives the derived molecular abundances. The upper limits to column densities and molecular abundances derived from nondetections of $\mathrm{SO}_{2}$ are listed in Table 4.
Figure 4 plots the variation of molecular abundances with bolometric temperature. The abundances of all three molecules show a tentative trend of decreasing abundance with increasing bolometric temperature. The mean fractional abundances, and uncertainty on the mean, for each molecule with detected emission, are listed in Table 5. The Class 0 sources have higher 
Table 3. continued.

\begin{tabular}{|c|c|c|c|c|c|c|c|}
\hline Source & Molecule & & $\begin{array}{r}\Delta v \\
\mathrm{~km} \mathrm{~s}^{-1}\end{array}$ & $\begin{array}{r}v \\
\mathrm{~km} \mathrm{~s}^{-1}\end{array}$ & $\begin{array}{r}\int T_{\mathrm{R}}^{*} \mathrm{~d} v \\
\mathrm{~K} \mathrm{~km} \mathrm{~s}^{-1}\end{array}$ & $\begin{array}{r}N_{\mathrm{mol}} \\
\times 10^{13} \mathrm{~cm}^{-2} \\
\end{array}$ & $\begin{array}{r}X_{\mathrm{mol}} \\
\times 10^{-9}\end{array}$ \\
\hline \multirow[t]{3}{*}{ TMR1 $(0,0)$} & $\mathrm{H}_{2} \mathrm{~S}_{1,0} \rightarrow 1_{0,1}$ & $*$ & $1.59(.21)$ & $6.24(.07)$ & $0.17(.02)$ & 0.37 & 0.45 \\
\hline & $\mathrm{C}^{18} \mathrm{O} 1 \rightarrow 0$ & $*$ & $0.58(.01)$ & $6.52(.00)$ & $1.39(.02)$ & 125.47 & \\
\hline & & & $0.40(.02)$ & $5.78(.01)$ & $0.32(.02)$ & 28.89 & \\
\hline \multirow[t]{3}{*}{$\operatorname{L} 1527(0,0)^{(a)}$} & $\mathrm{H}_{2} \mathrm{~S} 1_{1,0} \rightarrow 1_{0,1}$ & $*$ & $0.43(.07)$ & $5.98(.04)$ & $0.11(.01)$ & 0.24 & 0.32 \\
\hline & $\mathrm{SO} 4_{3} \rightarrow 3_{2}$ & $*$ & $0.41(.03)$ & $5.78(.01)$ & $0.17(.01)$ & 0.24 & 0.33 \\
\hline & $\mathrm{C}^{18} \mathrm{O} 1 \rightarrow 0$ & $*$ & $0.51(.01)$ & $6.00(.00)$ & $1.25(.02)$ & 112.83 & \\
\hline \multirow[t]{8}{*}{$\mathrm{S} 68 \mathrm{~N}(0,0)$} & $\mathrm{H}_{2} \mathrm{~S}_{1,0} \rightarrow 1_{0,1}$ & $*$ & $4.91(.26)$ & $8.28(.10)$ & $1.75(.08)$ & 3.81 & 1.42 \\
\hline & $\mathrm{SO} 4_{3} \rightarrow 3_{2}$ & $* *$ & $8.87(.68)$ & $6.67(.29)$ & $1.58(.11)$ & 2.26 & 8.02 \\
\hline & & * & $1.95(.04)$ & $8.48(.02)$ & $2.13(.20)$ & 3.05 & 1.14 \\
\hline & $\mathrm{SO}_{2} 5_{1,4} \rightarrow 4_{0,4}$ & $*$ & $3.95(.53)$ & $8.95(.20)$ & $0.23(.03)$ & 0.79 & 0.29 \\
\hline & & $*$ & $0.69(.11)$ & $8.45(.06)$ & $0.06(.01)$ & 0.21 & 0.08 \\
\hline & ${ }^{34} \mathrm{SO} 4_{3} \rightarrow 3_{2}$ & * & $0.98(.11)$ & 7.85(.04) & $0.14(.01)$ & 0.20 & 0.08 \\
\hline & $\mathrm{C}^{18} \mathrm{O} 1 \rightarrow 0$ & $*$ & $1.25(.01)$ & $8.47(.00)$ & $5.13(.04)$ & 463.61 & \\
\hline & & $* *$ & $0.63(.07)$ & 6.93(.03) & $0.32(.03)$ & 28.89 & \\
\hline \multirow[t]{2}{*}{$\operatorname{SMM} 8(0,0)$} & $\mathrm{H}_{2} \mathrm{~S}_{1,0} \rightarrow 1_{0,1}$ & & $2.67(.23)$ & $8.26(.08)$ & $0.96(.06)$ & 2.09 & .. \\
\hline & $\mathrm{SO} 4_{3} \rightarrow 3_{2}$ & & $1.52(.11)$ & $8.00(.04)$ & $0.72(.04)$ & 1.03 & .. \\
\hline S68N(339,146) & $\mathrm{H}_{2} \mathrm{~S}_{1,0} \rightarrow 1_{0,1}$ & & $3.71(.43)$ & $7.16(.19)$ & $0.43(.00)$ & 0.94 & .. \\
\hline \multirow[t]{2}{*}{ S68N(366,146) } & $\mathrm{H}_{2} \mathrm{~S}_{1,0} \rightarrow 1_{0,1}$ & & $1.34(.16)$ & 7.58(.07) & $0.42(.04)$ & 0.92 & .. \\
\hline & $\mathrm{SO} 4_{3} \rightarrow 3_{2}$ & & $0.88(.14)$ & 7.71(.06) & $0.24(.03)$ & 0.34 & .. \\
\hline \multirow[t]{2}{*}{ S68N(278,-7) } & $\mathrm{H}_{2} \mathrm{~S}_{1,0} \rightarrow 1_{0,1}$ & & $0.90(.10)$ & 7.60(.04) & $0.19(.02)$ & 0.41 & .. \\
\hline & $\mathrm{SO} 4_{3} \rightarrow 3_{2}$ & & $1.13(.12)$ & 7.51(.05) & $0.40(.03)$ & 0.57 & .. \\
\hline \multirow[t]{2}{*}{ S68N(143,190) } & $\mathrm{H}_{2} \mathrm{~S}_{1,0} \rightarrow 1_{0,1}$ & & $2.25(.14)$ & 7.51(.06) & $1.62(.08)$ & 3.53 & .. \\
\hline & $\mathrm{SO} 4_{3} \rightarrow 3_{2}$ & & $2.15(.07)$ & 7.72(.03) & $1.66(.05)$ & 2.38 & .. \\
\hline \multirow[t]{2}{*}{ S68N(-138,89) } & $\mathrm{H}_{2} \mathrm{~S}_{1,0} \rightarrow 1_{0,1}$ & & $3.45(.30)$ & $9.10(.14)$ & $0.43(.01)$ & 0.94 & .. \\
\hline & $\mathrm{SO} 4_{3} \rightarrow 3_{2}$ & & $2.49(.20)$ & $9.01(.07)$ & $0.85(.05)$ & 1.22 & .. \\
\hline \multirow[t]{6}{*}{$\operatorname{SMM} 11(0,0)$} & $\mathrm{H}_{2} \mathrm{~S}_{1,0} \rightarrow 1_{0,1}$ & & $1.72(.16)$ & 7.58(.05) & $0.66(.05)$ & 1.44 & .. \\
\hline & & & $1.00(.11)$ & $9.29(.05)$ & $0.28(.04)$ & 0.61 & .. \\
\hline & $\mathrm{SO} 4_{3} \rightarrow 3_{2}$ & & $1.48(.03)$ & $7.88(.02)$ & $2.13(.00)$ & 3.05 & .. \\
\hline & & & $0.86(.04)$ & $8.76(.02)$ & $0.68(.04)$ & 0.97 & .. \\
\hline & $\mathrm{SO}_{2} 5_{1,4} \rightarrow 4_{0,4}$ & & $0.81(.08)$ & $8.43(.03)$ & $0.16(.01)$ & 0.55 & .. \\
\hline & ${ }^{34} \mathrm{SO} 4_{3} \rightarrow 3_{2}$ & & $0.93(.06)$ & $7.63(.02)$ & $0.22(.01)$ & 0.32 & .. \\
\hline \multirow[t]{7}{*}{$\operatorname{SMM} 1(0,0)$} & $\mathrm{H}_{2} \mathrm{~S}_{1,0} \rightarrow 1_{0,1}$ & & $1.94(.17)$ & 7.63(.06) & $0.96(.07)$ & 2.09 & .. \\
\hline & & & $1.24(.13)$ & $9.56(.06)$ & $0.46(.06)$ & 1.00 & .. \\
\hline & $\mathrm{SO} 4_{3} \rightarrow 3_{2}$ & & $1.42(.09)$ & $9.04(.03)$ & $1.48(.06)$ & 2.12 & .. \\
\hline & & & $1.34(.05)$ & $7.86(.02)$ & $2.13(.04)$ & 3.05 & .. \\
\hline & $\mathrm{SO}_{2} 5_{1,4} \rightarrow 4_{0,4}$ & & $2.56(.30)$ & $8.14(.14)$ & $0.31(.04)$ & 1.06 & .. \\
\hline & & & $0.92(.08)$ & $8.66(.03)$ & $0.29(.04)$ & 1.00 & .. \\
\hline & ${ }^{34} \mathrm{SO} 4_{3} \rightarrow 3_{2}$ & & $1.25(.04)$ & $7.88(.02)$ & $0.53(.01)$ & 0.76 & .. \\
\hline \multirow[t]{4}{*}{$\operatorname{SMM} 4(0,0)$} & $\mathrm{H}_{2} \mathrm{~S}_{1,0} \rightarrow 1_{0,1}$ & & $2.26(.08)$ & 7.67(.03) & $1.62(.05)$ & 3.52 & .. \\
\hline & $\mathrm{SO} 4_{3} \rightarrow 3_{2}$ & & $1.86(.08)$ & 7.99(.03) & $1.92(.06)$ & 2.74 & .. \\
\hline & $\mathrm{SO}_{2} 5_{1,4} \rightarrow 4_{0,4}$ & & $0.53(.38)$ & $8.55(.07)$ & $0.04(.02)$ & 0.14 & .. \\
\hline & ${ }^{34} \mathrm{SO} 4_{3} \rightarrow 3_{2}$ & & $0.98(.15)$ & $7.10(.07)$ & $0.08(.01)$ & 0.12 & .. \\
\hline \multirow[t]{3}{*}{ B335 $(0,0)$} & $\mathrm{H}_{2} \mathrm{~S}_{1,0} \rightarrow 1_{0,1}$ & & $0.38(.14)$ & $8.52(.03)$ & $0.07(.02)$ & 0.15 & .. \\
\hline & & & $0.35(.09)$ & $8.02(.03)$ & $0.07(.02)$ & 0.15 & .. \\
\hline & $\mathrm{SO} 4_{3} \rightarrow 3_{2}$ & & $1.24(.26)$ & $8.42(.14)$ & $0.05(.01)$ & 0.36 &.. \\
\hline
\end{tabular}

${ }^{(a)}$ See text Sect. 3.1.

fractional abundances in $\mathrm{H}_{2} \mathrm{~S}$ and $\mathrm{SO}$ than the Class I sources. For $\mathrm{SO}_{2}$ there is a larger scatter in the points, but the nondetection of $\mathrm{SO}_{2}$ towards most of the Class I sources suggests this molecule may also have a higher fractional abundance towards Class 0 sources.

\subsection{Comparison with previous observations}

A comparison of the column densities calculated from these observations with previously published data from other telescopes suggests that emission from $\mathrm{H}_{2} \mathrm{~S}$ arises in clumps 
Table 3. continued.

\begin{tabular}{|c|c|c|c|c|c|c|c|}
\hline Source & Molecule & & $\begin{array}{r}\Delta v \\
\mathrm{~km} \mathrm{~s}^{-1}\end{array}$ & $\begin{array}{r}v \\
\mathrm{~km} \mathrm{~s}^{-1}\end{array}$ & $\int_{\mathrm{K} \mathrm{km} \mathrm{s}} T_{\mathrm{R}}^{*} \mathrm{~d} v$ & $\begin{array}{r}N_{\text {mol }} \\
\times 10^{13} \mathrm{~cm}^{-2}\end{array}$ & $\begin{array}{r}X_{\mathrm{mo}} \\
\times 10^{-9}\end{array}$ \\
\hline \multirow[t]{6}{*}{ L1152(0,0) } & $\mathrm{H}_{2} \mathrm{~S} 1_{1,0} \rightarrow 1_{0,1}$ & * & $0.68(.10)$ & $2.48(.04)$ & $0.11(.01)$ & 0.24 & 1.28 \\
\hline & $\mathrm{SO}_{4} \rightarrow 3_{2}$ & * & $0.48(.02)$ & $2.35(.01)$ & $0.33(.01)$ & 0.47 & 2.53 \\
\hline & & $* *$ & $0.32(.16)$ & $3.22(.06)$ & $0.03(.01)$ & 0.04 & 0.09 \\
\hline & $\mathrm{SO} 2_{2} \rightarrow 1_{1}$ & * & $0.67(.13)$ & $2.57(.07)$ & $0.04(.01)$ & 0.29 & 1.56 \\
\hline & $\mathrm{C}^{18} \mathrm{O} 1 \rightarrow 0$ & $* *$ & $0.77(.05)$ & $2.69(.04)$ & $0.71(.08)$ & 64.09 & \\
\hline & & * & $0.33(.09)$ & $2.44(.04)$ & $0.13(.08)$ & 11.73 & \\
\hline \multirow[t]{7}{*}{ L1157(0,0) } & $\mathrm{H}_{2} \mathrm{~S} 1_{1,0} \rightarrow 1_{0,1}$ & * & $1.14(.09)$ & $2.43(.03)$ & $0.30(.02)$ & 0.65 & 3.40 \\
\hline & $\mathrm{SO}_{4} \rightarrow 3_{2}$ & * & $5.54(.60)$ & $2.12(.21)$ & $0.59(.05)$ & 0.84 & 4.40 \\
\hline & & $* *$ & $0.69(.02)$ & $2.55(.01)$ & $0.71(.02)$ & 1.02 & 2.47 \\
\hline & $\mathrm{SO} 2_{2} \rightarrow 1_{1}$ & $* *$ & $0.98(.17)$ & $2.61(.05)$ & $0.12(.01)$ & 0.87 & 2.12 \\
\hline & ${ }^{34} \mathrm{SO} 4_{3} \rightarrow 3_{2}$ & * & $0.97(.16)$ & $1.99(.06)$ & $0.08(.01)$ & 0.12 & 0.60 \\
\hline & $\mathrm{C}^{18} \mathrm{O} 1 \rightarrow 0$ & $* *$ & $0.45(.04)$ & $2.74(.03)$ & $0.58(.08)$ & 52.35 & \\
\hline & & $*$ & $0.37(.14)$ & $2.32(.08)$ & $0.14(.08)$ & 12.64 & \\
\hline \multirow[t]{6}{*}{ L1262(0,0) } & $\mathrm{H}_{2} \mathrm{~S} 1_{1,0} \rightarrow 1_{0,1}$ & * & $1.81(.23)$ & $4.38(.09)$ & $0.19(.02)$ & 0.41 & 0.68 \\
\hline & $\mathrm{SO} 4_{3} \rightarrow 3_{2}$ & * & $0.63(.03)$ & $3.98(.01)$ & $0.31(.01)$ & 0.44 & 0.73 \\
\hline & $\mathrm{SO} 2_{2} \rightarrow 1_{1}$ & $*$ & $0.71(.10)$ & $4.20(.05)$ & $0.08(.01)$ & 0.58 & 0.95 \\
\hline & ${ }^{34} \mathrm{SO}_{3} \rightarrow 3_{2}$ & * & $0.46(.08)$ & $3.36(.04)$ & $0.04(.01)$ & 0.06 & 0.09 \\
\hline & $\mathrm{C}^{18} \mathrm{O} 1 \rightarrow 0$ & * & $0.58(.02)$ & $4.09(.01)$ & $0.98(.03)$ & 88.46 & \\
\hline & & & $0.22(.05)$ & $3.50(.02)$ & $0.08(.02)$ & 7.22 & \\
\hline
\end{tabular}
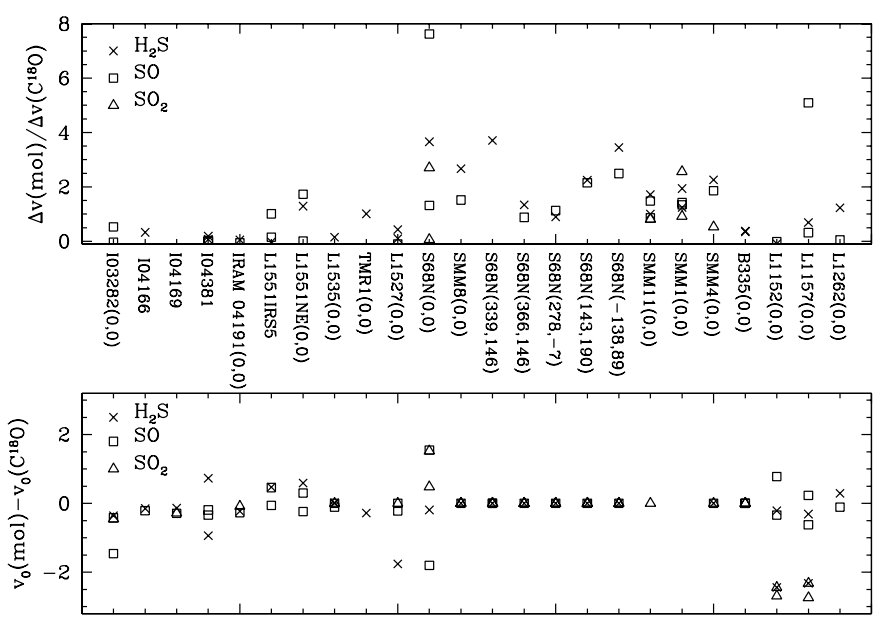

source (see top label)

Fig. 3. Top: variation of linewidth for the molecules detected towards each position, with respect to that of $\mathrm{C}^{18} \mathrm{O}$. Bottom: variation of line centre velocity for the molecules detected towards each position, with respect to that of $\mathrm{C}^{18} \mathrm{O}$. If a source has two components in a molecular line, the linewidths and velocities for both components have been plotted.

that are smaller than the $43^{\prime \prime}$ beamsize of the $12 \mathrm{~m}$ telescope. Table 6 lists the beamsize ratio and column density ratio for $\mathrm{H}_{2} \mathrm{~S}$ and $\mathrm{SO}$ towards L1157, and SO towards IRAS 04381+2540 (Bachiller \& Gutiêrrez 1997; Pratap et al. 1997).

Towards L1157, the ratio of $\mathrm{H}_{2} \mathrm{~S}$ column densities is similar to the ratio of the beam sizes, suggesting that emission from $\mathrm{H}_{2} \mathrm{~S}$ does not fill the beam, and so arises on scales smaller than the $17^{\prime \prime}$ beamsize of the IRAM $30 \mathrm{~m}$ telescope. Towards the same source, the ratio of SO column densities is
Table 4. Upper limits to integrated intensities, column densities and abundances from non-detections.

\begin{tabular}{llrrr}
\hline \hline Source & Molecule & $\begin{array}{r}\int T_{\mathrm{R}}^{*} \mathrm{~d} v \\
\mathrm{~km} \mathrm{~s}^{-1}\end{array}$ & $\begin{array}{r}N_{\text {mol }} \\
\times 10^{11}\end{array}$ & $\begin{array}{r}X_{\text {mol }} \\
\times 10^{-11}\end{array}$ \\
\hline I04166 & $\mathrm{SO}_{2}$ & 0.012 & 4.09 & 5.94 \\
L1551IRS5 & $\mathrm{SO}_{2}$ & 0.013 & 4.53 & 5.61 \\
L1551NE & $\mathrm{SO}_{2}$ & 0.012 & 4.04 & 4.11 \\
L1535 & $\mathrm{SO}_{2}$ & 0.011 & 3.75 & 5.89 \\
L1527 & $\mathrm{SO}_{2}$ & 0.011 & 3.94 & 5.28 \\
SMM8 & $\mathrm{SO}_{2}$ & 0.012 & 4.10 &.. \\
B335 & $\mathrm{SO}_{2}$ & 0.011 & 3.82 &.. \\
L1152 & $\mathrm{SO}_{2}$ & 0.013 & 4.35 & 6.55 \\
L1157 & $\mathrm{SO}_{2}$ & 0.013 & 4.39 & 7.27 \\
L1262 & $\mathrm{SO}_{2}$ & 0.013 & 4.49 & 5.81 \\
\hline
\end{tabular}

Table 5. Mean fractional abundances for molecules detected towards core positions. Errors on the means are given in parentheses. For $\mathrm{SO}_{2}$ the class I category has only one detected point. The remainder of the class I sources have fractional abundances of $<0.5 \times 10^{-10}$.

\begin{tabular}{lrr}
\hline \hline & \multicolumn{2}{c}{ Fractional Abundance $\left(\times 10^{-10}\right)$} \\
Molecule & Class 0 cores & Class I cores \\
\hline $\mathrm{H}_{2} \mathrm{~S}$ & $18.3(5.7)$ & $7.9(1.5)$ \\
$\mathrm{SO}$ & $31.3(9.3)$ & $7.1(2.2)$ \\
$\mathrm{SO}_{2}$ & $4.1(2.1)$ & $3.0(.)$. \\
\hline
\end{tabular}

larger than the beamsize ratio, suggesting that the emission is more extended in this molecule, filling the $30 \mathrm{~m}$ beam, but not the $12 \mathrm{~m}$ beam. A similar comparison of SO observations towards IRAS $04381+2540$ suggests that SO emission is extended on scales greater than the $43^{\prime \prime}$ beamsize of the $12 \mathrm{~m}$ towards this source. Towards L1157 (Bachiller et al. 2001), where we did not detect $\mathrm{SO}_{2}$, emission from $\mathrm{SO}_{2}$ arises in small 
Table 6. A comparison of with observations undertaken at different telescopes. The ratio columns list the ratio with our observations.

\begin{tabular}{rrrrrrr}
\hline \hline Source & Telescope & Molecule & Beamsize Ratio & Column Density Ratio & Abundance Ratio & Reference \\
\hline L1157 & IRAM 30 m & $\mathrm{H}_{2} \mathrm{~S}$ & 0.16 & 0.13 & 0.31 & 1 \\
L1157 & IRAM 30 m & SO & 0.16 & 0.93 & 1.37 & 1 \\
04381 & FCRAO & SO & 0.75 & 1.13 &.. & 2 \\
\hline
\end{tabular}

1. (Bachiller \& Gutiêrrez 1997).

2. (Pratap et al. 1997).
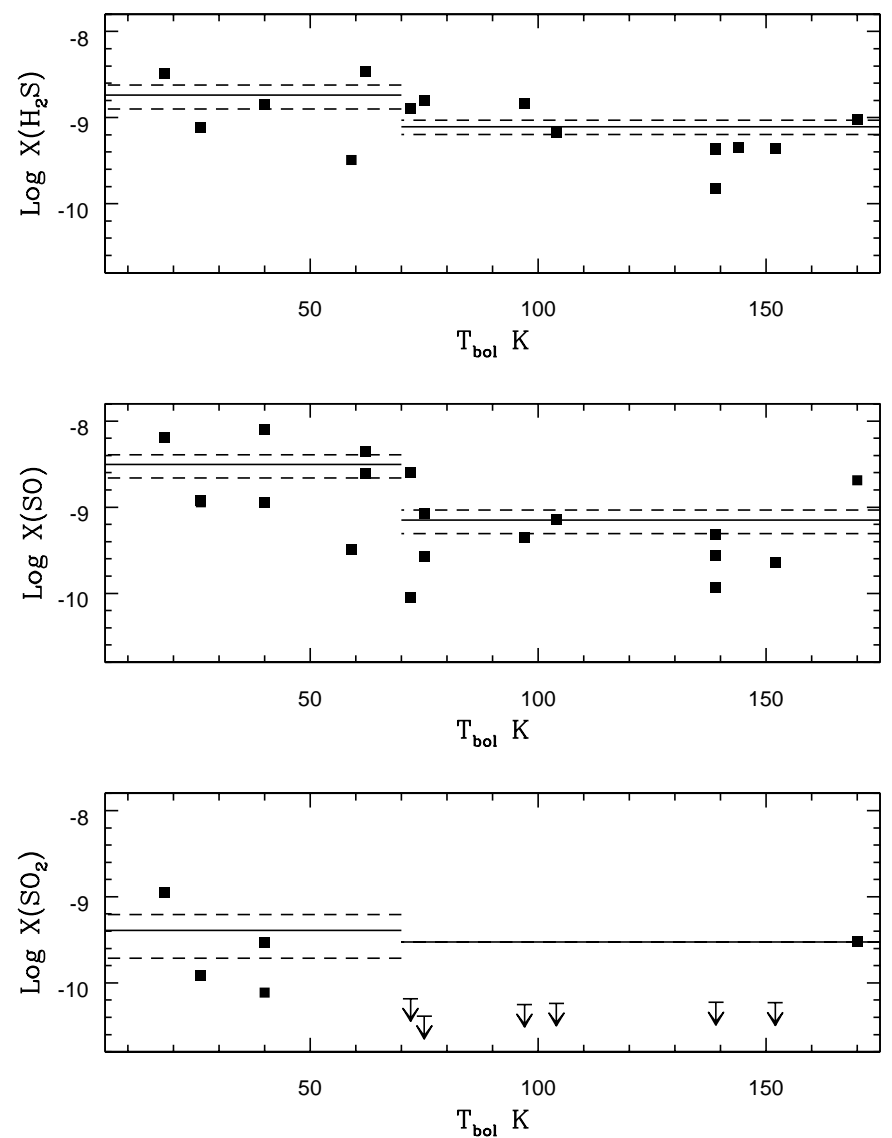

Fig. 4. Variation of abundance with $T_{\text {bol }}$ towards the observed core positions. Maximum values from non-detections are marked as upper limits. The solid lines mark the mean abundance (excluding nondetections) for the Class 0 sources $\left(T_{\text {bol }} \leq 70 \mathrm{~K}\right)$ and the Class I sources $\left(T_{\mathrm{bol}}>70 \mathrm{~K}\right)$. Dashed lines indicate the errors on the means.

clumps within the outflow, in the same positions where emission from molecules such as $\mathrm{CO}, \mathrm{SO}, \mathrm{CH}_{3} \mathrm{OH}$ and $\mathrm{HCN}$ peak.

These comparisons suggest that we may be underestimating the abundance of $\mathrm{H}_{2} \mathrm{~S}$ and $\mathrm{SO}_{2}$ towards protostellar sources, since the emission is likely to arise in smaller regions than is traced by emission from $\mathrm{C}^{18} \mathrm{O}$. However since there is little detailed information at sufficiently high angular resolution on the spatial distribution of the emission towards the sources we have observed, for the purposes of analysis we assume that the emission from each species fills the beam and originates from the same gas. The effects of these assumptions on the modelling results are discussed in Sect. 6.

\subsection{Origin of the emission}

The $\mathrm{H}_{2} \mathrm{~S}$ abundances towards the sources, while not as high as in hot cores (Hatchell et al. 1998), are nevertheless inconsistent with gas phase formation of the molecule. Millar \& Herbst (1990) showed that models of the gas phase chemistry in TMC1 produced an abundance of $\mathrm{H}_{2} \mathrm{~S}$ of $\sim 6 \times 10^{-11}$, about 10 times less than observed in TMC-1 and the sources studied here. Gas phase models fail by an even larger margin for the apparently quiescent core L134N where observations by Minh et al. (1989) show it to have an $\mathrm{H}_{2} \mathrm{~S}$ column density and abundance similar to the sources observed here. The difficulty in producing sufficient $\mathrm{H}_{2} \mathrm{~S}$ in the gas phase led to Charnley (1997) and Millar et al. (1997) suggesting that in hot cores it is the release of $\mathrm{H}_{2} \mathrm{~S}$ from grain mantles that produces the observed gas phase abundance of this species. Subsequent to its release the $\mathrm{H}_{2} \mathrm{~S}$ drives a chemistry which produces $\mathrm{SO}$ and $\mathrm{SO}_{2}$ and ultimately $\mathrm{CS}$ and $\mathrm{H}_{2} \mathrm{CS}$. It was a chemical model of this release and processing of $\mathrm{H}_{2} \mathrm{~S}$ which Hatchell et al. (1998) used together with observations of $\mathrm{H}_{2} \mathrm{~S}, \mathrm{SO}$ and $\mathrm{SO}_{2}$ towards hot cores to provide a chemical clock to determine the time since the grains were heated sufficiently to release the $\mathrm{H}_{2} \mathrm{~S}$.

In contrast to $\mathrm{H}_{2} \mathrm{~S}$, column densities of $\mathrm{SO}$ (and possibly $\mathrm{SO}_{2}$ ) consistent with the observed column densities can be produced by gas phase reactions. Models show that significant abundances of both $\mathrm{SO}$ and $\mathrm{SO}_{2}$ are formed in in the late stages (ages $>10^{5} \mathrm{yr}$ ) of the chemical evolution of a dark cloud (Dickens et al. 2000; Pratap et al. 1997), although even at this stage the predicted $\mathrm{SO}_{2}$ abundance is significantly less than observed (Dickens et al. 2000). On the other hand Chernin et al. (1994) mapped the SO emission from outflow sources and although based on the linewidths claimed that the emission was from quiescent gas, they also noted that in two sources, L1551 and IRAS $03282+3035$, the SO appears to be concentrated at the edge of the outflow cavities. Inspection of their maps show this to be also true in L1448. Similarly, detailed maps of L1157 by Bachiller et al. (2001) show the $\mathrm{SO}$ and $\mathrm{SO}_{2}$ emission to be associated with the outflow from the source.

The relatively narrow linewidths of much of the SO emission, both in Chernin et al. (1994) and in this work, (and that of $\mathrm{H}_{2} \mathrm{~S}$ and $\mathrm{SO}_{2}$ ) do not necessarily indicate that the $\mathrm{SO}$ is associated with undisturbed cloud material. Buckle \& Fuller (2002) have shown that towards Class 0 and I sources even velocity components with widths similar to those seen in the SO and $\mathrm{SO}_{2}$ have an enhanced methanol abundance consistent with the material being disturbed and grain mantles being released. This is consistent with correlation between the linewidth of 

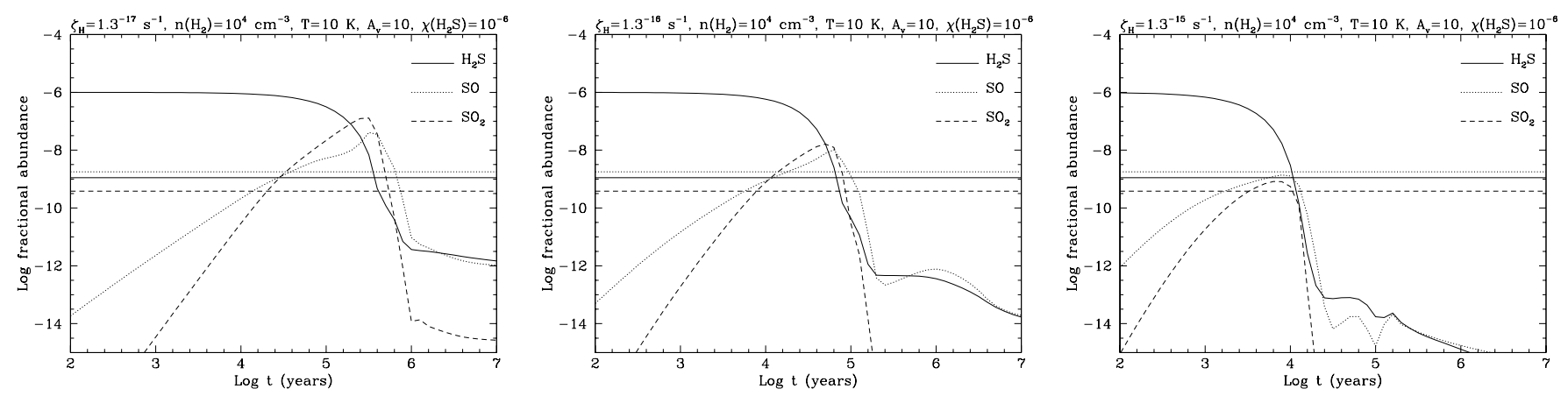

Fig. 5. Chemical model: variations with $\zeta_{\mathrm{H}}$. The evolution of fractional abundance is shown in bold lines for $\mathrm{H}_{2} \mathrm{~S}$ (solid), $\mathrm{SO}$ (dotted) and $\mathrm{SO}_{2}$ (dashed). Additionally, the average abundance derived from the observational data for the three molecules is indicated by the lighter horizontal lines, with the same line type used for each molecule. The important initial parameters of each run of the model are displayed at the top of each plot. $T_{\text {kinetic }}=10 \mathrm{~K}, n\left(\mathrm{H}_{2}\right)=10^{4} \mathrm{~cm}^{-3}$, and $\chi\left(\mathrm{H}_{2} \mathrm{~S}\right)=10^{-6}$ are fixed parameters for the models in this plot.

"quiescent" material and outflowing material in a sample of Taurus sources found by Fuller \& Ladd (2002).

Therefore although modelling indicates that the $\mathrm{SO}$, and to a lesser extent $\mathrm{SO}_{2}$, could be produced in quiescent gas, the mapping evidence suggests that in regions with outflows much of the emission is associated with outflow activity. Given this, the probable grain mantle origin of the $\mathrm{H}_{2} \mathrm{~S}$, and the potential importance of a chemical clock in classifying and studying the evolution of low mass protostars, we chose to model our observations using the same model as Hatchell et al. (1998) used to model hot cores sources. In doing this we will ignore routes forming $\mathrm{SO}$ and $\mathrm{SO}_{2}$ other than through $\mathrm{H}_{2} \mathrm{~S}$. The net effect of this is that the timescales we derive are larger than would be derived if other sources of $\mathrm{SO}$ and $\mathrm{SO}_{2}$ were included.

\section{Chemical clocks}

The chemical model, based on models of hot core chemistry developed by Millar et al. (1997) and Charnley (1997) and then updated by Hatchell et al. (1998), is fully described in these papers, and only briefly outlined here. The model, which includes over 200 species, calculates the abundances as a function of time for a parcel of gas with the initial temperature, density, grain mantle species abundances and cosmic ray ionisation rate as input parameters. After the initial release of $\mathrm{H}_{2} \mathrm{~S}$ into the gas phase as grain mantles are evaporated, destruction of this molecule via $\mathrm{H}$ and $\mathrm{H}_{3} \mathrm{O}^{+}$produces sulphur in the form of $\mathrm{S}$ or $\mathrm{H}_{3} \mathrm{~S}^{+}$. Following reactions with $\mathrm{OH}$ and $\mathrm{O}_{2}$, the species $\mathrm{SO}$ and then $\mathrm{SO}_{2}$ are formed. The abundances of these daughter products falls at later times, and most of the sulphur is converted into the form of $\mathrm{CS}, \mathrm{H}_{2} \mathrm{CS}$ and OCS. Since no grain chemistry is included in the model, the initial estimates for grain-evaporated species are assumed from consideration of observations of ices and molecules in the gas phase. In the models described here, we use a number of conditions appropriate to low mass cores, such as lower temperatures and densities, in order to test whether the model for hot cores is also applicable to their low mass counterparts, and whether the models produce useful age constraints.

\subsection{Results from the model}

As initial input parameters to the model, we consider total hydrogen densities of $10^{3}$ to $10^{6} \mathrm{~cm}^{-3}$ and temperatures between 5 and $100 \mathrm{~K}$. We vary the cosmic ray ionisation rate, $\zeta_{\mathrm{H}}$, between $1.3 \times 10^{-17} \mathrm{~s}^{-1}$, the standard interstellar value, and enhanced rates of $1.3 \times 10^{-16} \mathrm{~s}^{-1}$ and $1.3 \times 10^{-15} \mathrm{~s}^{-1}$. The results of the model for various values of the input parameters are shown in Figs. 5 to 8.

The plots shown in Figs. 5 to 8 display the predicted abundances of $\mathrm{H}_{2} \mathrm{~S}$, SO and $\mathrm{SO}_{2}$ as a function of time (bold lines). The evaporation of $\mathrm{H}_{2} \mathrm{~S}$ from the grain mantles is modelled by an initially high abundance of this molecule. As the chemical network proceeds, the abundance of $\mathrm{H}_{2} \mathrm{~S}$ falls, and the abundances of $\mathrm{SO}$ and $\mathrm{SO}_{2}$ rise. In discussing the variation of the chemistry with input parameters, a convenient point in the predicted chemical evolution to compare is the point at which the abundance of $\mathrm{H}_{2} \mathrm{~S}$ has dropped to $1 \%$ of its initial value. In most cases, this is also the point at which the abundances of $\mathrm{SO}$ and $\mathrm{SO}_{2}$ reach their peak. Also shown on the plots are the average abundances derived from the observational data for the three molecules (lighter horizontal lines). The line type used for each molecule is the same for the predicted and observed abundances. A model which fits the abundances should intersect the observed abundances for all three molecules at the same point in time.

Increasing the cosmic ionisation rate by a factor of 100 , from the standard rate of $1.3 \times 10^{-17} \mathrm{~s}^{-1}$ to $1.3 \times 10^{-15} \mathrm{~s}^{-1}$, increases the rate at which the reactions proceed (Fig. 5). At $\zeta_{\mathrm{H}}=1.3 \times 10^{-17} \mathrm{~s}^{-1}, \mathrm{H}_{2} \mathrm{~S}$ drops to $1 \%$ of its initial value at $2 \times$ $10^{5}$ years, with $\mathrm{SO}$ and $\mathrm{SO}_{2}$ reaching their peak $\sim 2 \times 10^{5}$ years later. At $\zeta_{\mathrm{H}}=1.3 \times 10^{-15} \mathrm{~s}^{-1}, \mathrm{H}_{2} \mathrm{~S}$ drops to $1 \%$ of its initial value at $6 \times 10^{3}$ years, with $\mathrm{SO}$ and $\mathrm{SO}_{2}$ reaching their peak $\sim 2 \times 10^{3}$ years later. Higher cosmic ray ionisation rates lead to higher ionisation fractions, resulting in a more active, faster, ion-molecule chemistry (Millar et al. 1997).

The abundances of $\mathrm{H}_{2} \mathrm{~S}, \mathrm{SO}$ and $\mathrm{SO}_{2}$ approximately scale with the initial abundance of $\mathrm{H}_{2} \mathrm{~S}$ (Fig. 6). With an initial $\mathrm{H}_{2} \mathrm{~S}$ fractional abundance of $10^{-6}, \mathrm{H}_{2} \mathrm{~S}$ drops to $1 \%$ of its initial value at $3 \times 10^{4}$ years, with $\mathrm{SO}$ and $\mathrm{SO}_{2}$ reaching their peak abundances, of $\sim 10^{-8}, 3 \times 10^{4}$ years later. With an initial $\mathrm{H}_{2} \mathrm{~S}$ fractional abundance of $10^{-9}$, the timescale of the 

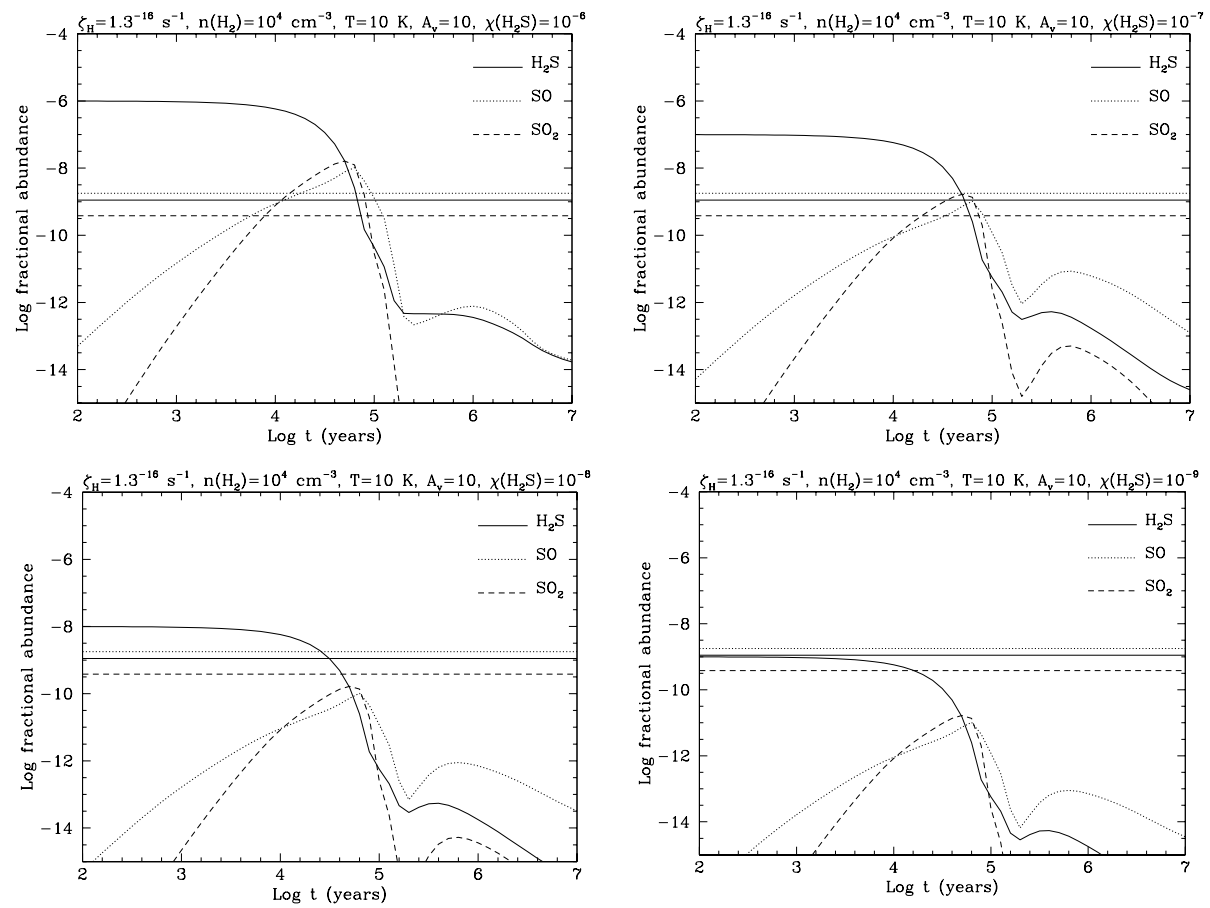

Fig. 6. Chemical model: as Fig. 5 , showing variations with $\mathrm{H}_{2} \mathrm{~S}$ initial abundance. $T_{\text {kinetic }}=10 \mathrm{~K}, n\left(\mathrm{H}_{2}\right)=10^{4} \mathrm{~cm}^{-3}$ and $\zeta_{\mathrm{H}}=1.3 \times 10^{-16}$ are fixed parameters for the models in this plot.

chemical evolution remains the same, but $\mathrm{SO}$ and $\mathrm{SO}_{2}$ peak abundances are $\sim 10^{-11}$.

Figure 7 displays the variations in molecular abundances as the total hydrogen density is increased. As the density is increased, the peak abundances of $\mathrm{SO}$ and $\mathrm{SO}_{2}$ rise. In addition, the peak abundance of $\mathrm{SO}_{2}$ rises above that of $\mathrm{SO}$ for the higher densities, until all three abundances start to fall. Thereafter, the $\mathrm{SO}_{2}$ abundance drops more steeply than the $\mathrm{SO}$ or $\mathrm{H}_{2} \mathrm{~S}$ abundance. Increased densities also drive the reactions forward at a faster rate. At $10^{3} \mathrm{~cm}^{-3}$, with $T=10 \mathrm{~K}$ and $\zeta_{\mathrm{H}}=1.3 \times 10^{-16} \mathrm{~s}^{-1}$ the $\mathrm{H}_{2} \mathrm{~S}$ abundance falls to $1 \%$ of its initial value after $7.9 \times 10^{4}$ years; at $10^{6} \mathrm{~cm}^{-3}$, the $1 \%$ value is reached after $3.5 \times 10^{4}$ years.

Varying the kinetic temperature between 5 and $100 \mathrm{~K}$ makes no significant difference to the timescale of the chemical evolution (Fig. 8), with the $\mathrm{H}_{2} \mathrm{~S}$ abundance falling to $1 \%$ of its value after $3 \times 10^{4}$ years, if $n\left(\mathrm{H}_{2}\right)=10^{4} \mathrm{~cm}^{-3}$ and $\zeta_{\mathrm{H}}=1.3 \times 10^{-16} \mathrm{~s}^{-1}$. As the kinetic temperature increases above $20 \mathrm{~K}$, the peak abundances of both $\mathrm{SO}$ and $\mathrm{SO}_{2}$ increase by a factor $\sim 3$ at $40 \mathrm{~K}$, and by a factor of 10 at $100 \mathrm{~K}$.

\section{Matching models and observations}

The observations that we have of sulphur bearing species in low mass cores suggests that these molecules are formed in a relatively low temperature environment. The higher lying SO $J_{N}=2_{2} \rightarrow 1_{1}$ transition was only marginally detected towards $25 \%$ of sources $\left(T_{\mathrm{R}}^{*}<0.3 \mathrm{~K}\right)$ although the lower lying SO $J_{N}=4_{3} \rightarrow 3_{2}$ transition has peak intensities up to $2.5 \mathrm{~K}$. Our observations, in agreement with other authors (see van Dishoeck \& Blake 1998, and references therein), suggest that low mass cores do not reach the high temperatures, of several hundred Kelvin, that have been derived for high mass cores
(Kurtz et al. 2000), except in small, shocked regions surrounding the outflow (Langer et al. 2000). Low mass cores are also lower density $\left(n\left(\mathrm{H}_{2}\right) \sim 10^{4}-10^{5} \mathrm{~cm}^{-3}\right.$ (Langer et al. 2000) than high mass cores, which typically have $n\left(\mathrm{H}_{2}\right) \geq 10^{7} \mathrm{~cm}^{-3}$ (Kurtz et al. 2000).

The observed range in abundances of all three molecules differs by less than two orders of magnitude (Fig. 4), ranging from a few $\times 10^{-11}$, to a few $\times 10^{-9}$ over the whole sample. In order to keep the predicted abundances from the model within this range $\left(\sim 10^{-10}\right)$, the model requires an initial $\mathrm{H}_{2} \mathrm{~S}$ abundance $>10^{-8} \mathrm{~cm}^{-2}$.

The similarity of the observed fractional abundances for the three molecules rules out a very early or very late chemical evolutionary age for the observed sources. Since the observed abundances of $\mathrm{H}_{2} \mathrm{~S}$ are similar to the observed abundances of $\mathrm{SO}$ and $\mathrm{SO}_{2}$, the sources are past the chemical evolutionary stage where the $\mathrm{H}_{2} \mathrm{~S}$ abundance falls to $1 \%$ of its initial value. In many of the models, the abundance of $\mathrm{SO}_{2}$ peaks at a higher value than the abundance of SO; after the peak, the abundance of $\mathrm{SO}_{2}$ falls more steeply than the abundance of $\mathrm{SO}$ or $\mathrm{H}_{2} \mathrm{~S}$. The low observed abundances of $\mathrm{SO}_{2}$, and the absence of emission from this molecule towards many of the sources, suggest that the sources are past the chemical evolutionary stage where the abundances of $\mathrm{SO}$ and $\mathrm{SO}_{2}$ peak.

At very late chemical evolutionary stages, where the predicted abundances fall much less rapidly, the predicted abundances of the three molecules are too low to provide a match to the observed abundances, even with the highest initial $\mathrm{H}_{2} \mathrm{~S}$ abundance (Fig. 6). The data we have, when compared to the chemical clock model, suggests that the sources are at a chemical evolutionary stage where the abundances of all three molecules are falling relatively quickly. 

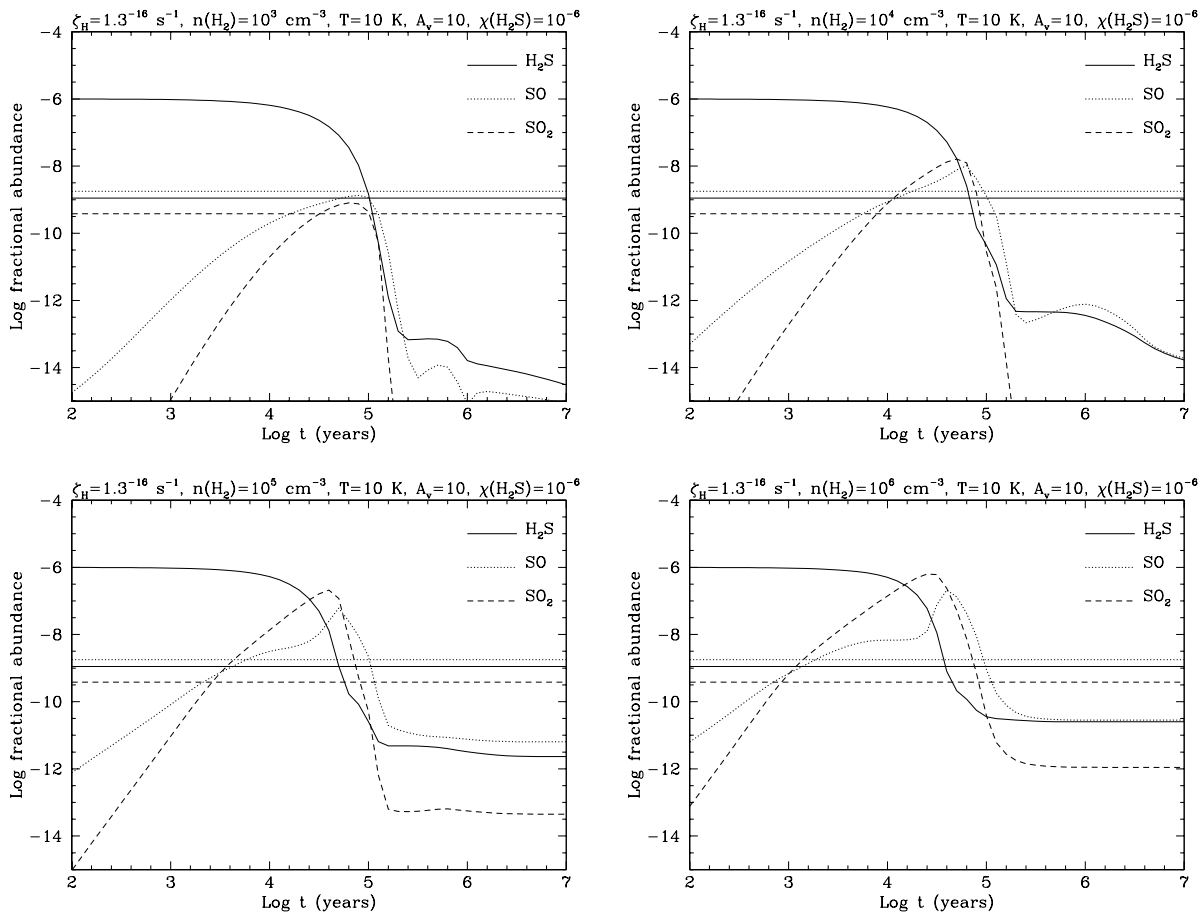

Fig. 7. Chemical model: as Fig. 5, showing variations with $\mathrm{H}_{2}$ density. $T_{\text {kinetic }}=10 \mathrm{~K}, \chi\left(\mathrm{H}_{2} \mathrm{~S}\right)=10^{-6}$ and $\zeta_{\mathrm{H}}=1.3 \times 10^{-16}$ are fixed parameters for the models in this plot.

The point at which this stage of the chemical evolution occurs depends upon the input parameters. For the highest cosmic ionisation rates (Fig. 5), this stage occurs at $\sim 10^{4}$ years, and lasts for a few $\times 10^{4}$ years. With lower cosmic ionisation rates, this stage occurs later, $\sim 10^{6}$ years, and lasts for a longer time. The timescale of the chemical evolution is heavily dependent upon the value of $\zeta_{\mathrm{H}}$ used, and could be made shorter or longer by respectively increasing or decreasing $\zeta_{\mathrm{H}}$ by larger factors. Using the standard interstellar value for the cosmic ionisation rate $\left(\zeta_{\mathrm{H}}=1.3 \times 10^{-17} \mathrm{~s}^{-1}\right)$ provides timescales $\left(\sim 10^{6} \mathrm{yr}\right)$ that are too long for these sources (Fig. 5) compared to other estimates (e.g. Mundy et al. 2000). In order to match the observed abundances, and the generally accepted ages of low mass protostellar sources, we need to use the enhanced rates of $\zeta_{\mathrm{H}}$ for the model.

Recent evidence for a higher than standard cosmic ionization rate $\left(\zeta_{\mathrm{H}}=1.3 \times 10^{-17} \mathrm{~s}^{-1}\right)$ in dense clouds comes from modelling the chemistry of dark clouds (Dickens et al. 2000; Pratap et al. 1997), where $\zeta_{\mathrm{H}}>6 \times 10^{-17} \mathrm{~s}^{-1}$ is need to reproduce the observed abundances and from detailed studies of the molecular ionization fraction (de Boisanger et al. 1996; van Dishoeck \& Black 1986) which require $\zeta_{\mathrm{H}}>10^{-16} \mathrm{~s}^{-1}$ and possibly as high as $3 \times 10^{-15} \mathrm{~s}^{-1}$.

In summary, Figs. 5 to 8 show that the chemical evolution model of sulphur-bearing species, originally developed for high mass cores, is able to provide a reasonable simultaneous match to the average abundances of $\mathrm{H}_{2} \mathrm{~S}, \mathrm{SO}$ and $\mathrm{SO}_{2}$, of $10^{-9}$ to $10^{-11}$, for the lower temperature and density conditions that are applicable to low mass cores, provided that, initially, $\chi\left(\mathrm{H}_{2} \mathrm{~S}\right)>10^{-8}$, and $\zeta_{\mathrm{H}}>1.3 \times 10^{-17} \mathrm{~s}^{-1}$. For these parameters, the timescale at which the model abundances are consistent with the observed abundances range from $10^{4}-10^{5} \mathrm{yr}$. The model provides matches to our low mass sources that fit at least as well as to the high mass hot cores modelled by Hatchell et al. (1998).

\subsection{Evolutionary trends}

The chemical model predicts varying abundances for these three molecules at different evolutionary ages. Initially, the $\mathrm{H}_{2} \mathrm{~S}$ abundance is much higher than that of $\mathrm{SO}$ or $\mathrm{SO}_{2}$. For a period of a few $\times 10^{4}$ to a few $\times 10^{5}$ years (dependent upon the model parameters), the abundances remain within an order of magnitude of each other, which matches the general pattern of observed abundances. During this time, SO becomes the most abundant molecule, followed by $\mathrm{H}_{2} \mathrm{~S}$, and then $\mathrm{SO}_{2}$. It is this period of the evolutionary timescale which most closely matches our data. Thereafter, the abundances of all three molecules drop to values several orders of magnitude below the observed abundances.

One clear trend evident in the data is the non-detection of $\mathrm{SO}_{2}$ towards any of the Class I sources except one, whereas it is detected towards 6 out of the 10 Class 0 sources (Table 5). Within the timescale range where we can match model data to observational data the chemical clock model predicts that the abundance of $\mathrm{SO}_{2}$ falls more steeply with time than the abundances of $\mathrm{H}_{2} \mathrm{~S}$ or $\mathrm{SO}$, so this difference is consistent with the Class I sources being older than the Class 0 sources. Therefore, overall the observational data, compared to predicted abundances from the chemical clock model, suggests that Class I sources are at a later chemical evolutionary stage than their Class 0 counterparts. Indeed the trend for the Class 0 sources to have higher molecular abundances is also evident in $\mathrm{H}_{2} \mathrm{~S}$ and SO (Table 5). 

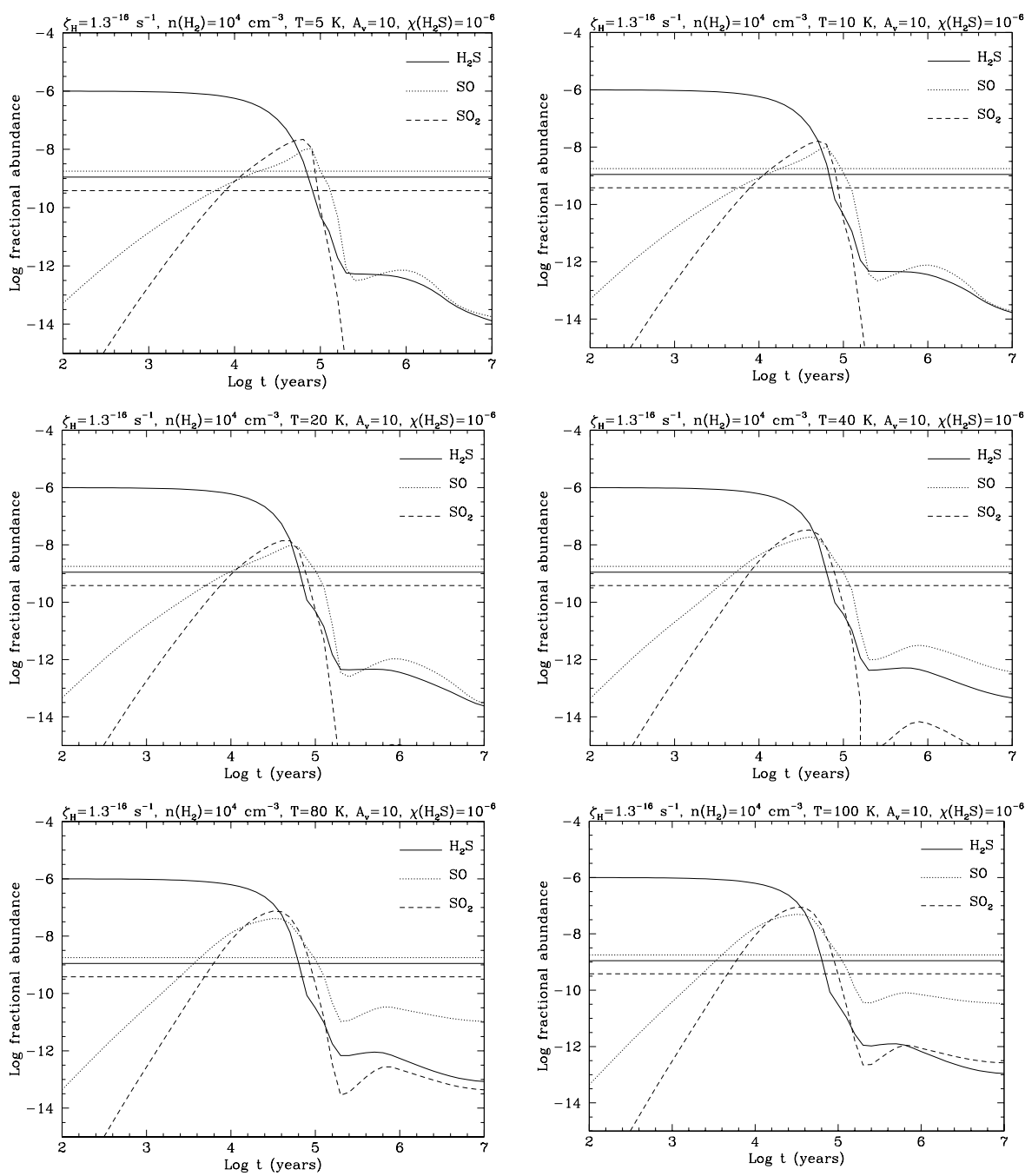

Fig. 8. Chemical model: as Fig. 5 , showing variations with kinetic temperature. $n\left(\mathrm{H}_{2}\right)=10^{4} \mathrm{~cm}^{-3}, \zeta_{\mathrm{H}}=1.3 \times 10^{-16}$ and $\chi\left(\mathrm{H}_{2} \mathrm{~S}\right)=10^{-6}$ are fixed parameters for the models in this plot.

In Fig. 9, the best model matches to the average Class 0 abundances (top) and Class I abundances (bottom) have been plotted. As can be seen from the plot, the chemical model suggests that the Class I sources must be at a chemical stage that is at least $3 \times 10^{3}$ years later than the Class 0 sources, or $30 \%$ more than the canonical Class 0 age.

\subsection{Individual sources}

Towards four of the sources that we observed, IRAS 03282+3035, IRAM 04191+1522, S68N (all Class 0 sources) and IRAS 04169+2702 (Class I), we have detected all three of the sulphur-bearing species, and have $\mathrm{C}^{18} \mathrm{O}$ observations that enable us to derive abundance estimates. Figure 10 plots the column densities and abundance estimates derived towards these sources. The $x$-axis gives the name of the source, with the three Class 0 sources plotted first, then the Class I source.

The abundances for each molecule towards these sources varies by only a factor of a few between the sources. The largest difference is between S68N and the other three sources. S68N has much higher abundances of $\mathrm{H}_{2} \mathrm{~S}$ and $\mathrm{SO}_{2}$ than the other sources, although the abundance of $\mathrm{SO}$ does not show such a large difference. Given the density of very young sources in the Serpens star forming cluster, it is perhaps not surprising that $\mathrm{S} 68 \mathrm{~N}$ is distinguishable from the other three sources, which have formed in more isolated environments. Due to the similar abundances, the sources are all reasonably matched by the same models. In order to define the best match to a source, we use a simple difference $\chi^{2}$ test:

$$
\begin{aligned}
\chi^{2}= & \frac{1}{3}\left(\left(\frac{X_{d}\left(\mathrm{H}_{2} \mathrm{~S}\right)-X_{m}\left(\mathrm{H}_{2} \mathrm{~S}\right)}{X_{d}\left(\mathrm{H}_{2} \mathrm{~S}\right)}\right)^{2}+\left(\frac{X_{d}(\mathrm{SO})-X_{m}(\mathrm{SO})}{X_{d}(\mathrm{SO})}\right)^{2}\right. \\
& \left.+\left(\frac{X_{d}\left(\mathrm{SO}_{2}\right)-X_{m}\left(\mathrm{SO}_{2}\right)}{X_{d}\left(\mathrm{SO}_{2}\right)}\right)^{2}\right)^{1 / 2}
\end{aligned}
$$

where $X_{\mathrm{d}(\mathrm{mol})}, X_{\mathrm{m}(\mathrm{mol})}$ refer to the observed and model abundances respectively. Using this $\chi^{2}$ test, the best match to each of the sources is provided by the model parameters listed in Table 7, and plotted in Fig. 11. The matches to the data are characterised by high cosmic ray ionisation rates, low volume 

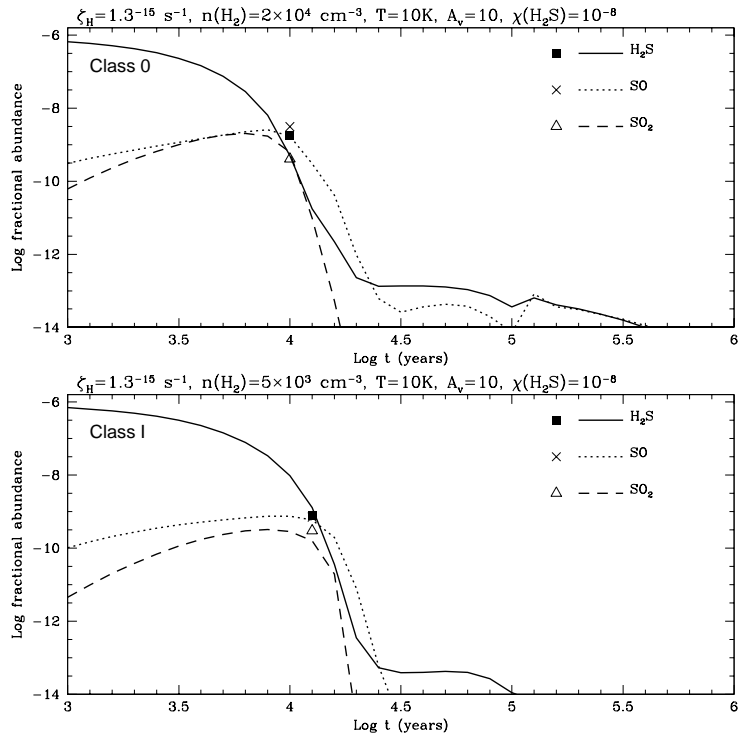

Fig. 9. A comparison of the predicted abundances from models which best match the Class 0 average abundances (top), and the Class I average abundances (bottom). The Class I sources have very similar $\mathrm{H}_{2} \mathrm{~S}$ and $\mathrm{SO}$ abundances and the markers in the figure are nearly coincident.
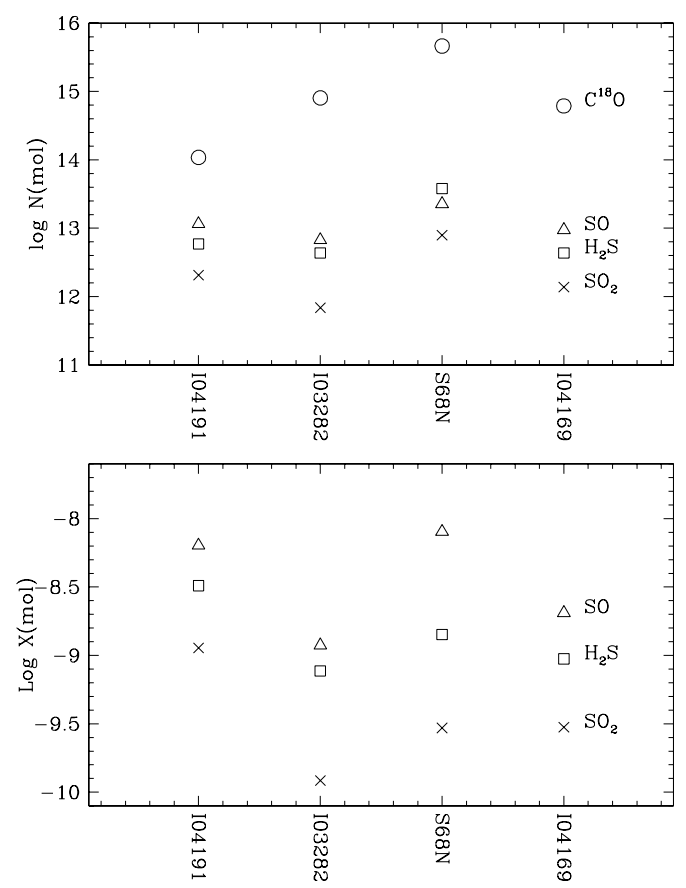

Fig. 10. The abundances and column densities for sulphur-bearing species in four sources where $\mathrm{H}_{2} \mathrm{~S}, \mathrm{SO}, \mathrm{SO}_{2}$ and $\mathrm{C}^{18} \mathrm{O}$ were detected.

densities, and early timescales. Models with lower cosmic ray ionisation rates, approaching the standard value of $1.3 \times$ $10^{-17} \mathrm{~s}^{-1}$, provide significantly worse matches to the data. Since we are unable to provide constraints on the cosmic ionisation rates towards these sources, and additionally, the cosmic ray ionisation rate has a large effect on the timescale for chemical evolution, for these fits we have constrained the fit so that $\zeta_{\mathrm{H}}=1.3 \times 10^{-15} \mathrm{~s}^{-1}$.

Volume densities below $10^{5} \mathrm{~cm}^{-3}$ provide the best matches to the data; above $10^{5} \mathrm{~cm}^{-3}$, the $\chi^{2}$ test suggests significantly
Table 7. Parameters of model matches to observational data.

\begin{tabular}{lrrrr}
\hline \hline Parameter & $\mathrm{I} 04191$ & $\mathrm{I} 03282$ & $\mathrm{~S} 68 \mathrm{~N}$ & $\mathrm{I} 04169$ \\
\hline$\zeta_{\mathrm{H}}\left(\mathrm{s}^{-1}\right)$ & $1.3 \times 10^{-15}$ & $1.3 \times 10^{-15}$ & $1.3 \times 10^{-15}$ & $1.3 \times 10^{-15}$ \\
$n\left(\mathrm{H}_{2}\right)\left(\mathrm{cm}^{-3}\right)$ & $5 \times 10^{3}$ & $5 \times 10^{3}$ & $5 \times 10^{3}$ & $5 \times 10^{3}$ \\
$T_{K}(\mathrm{~K})$ & 50 & 10 & 50 & 10 \\
Time $(\mathrm{yrs})$ & $1.3 \times 10^{4}$ & $1.3 \times 10^{4}$ & $1.3 \times 10^{4}$ & $1.3 \times 10^{4}$ \\
\hline
\end{tabular}
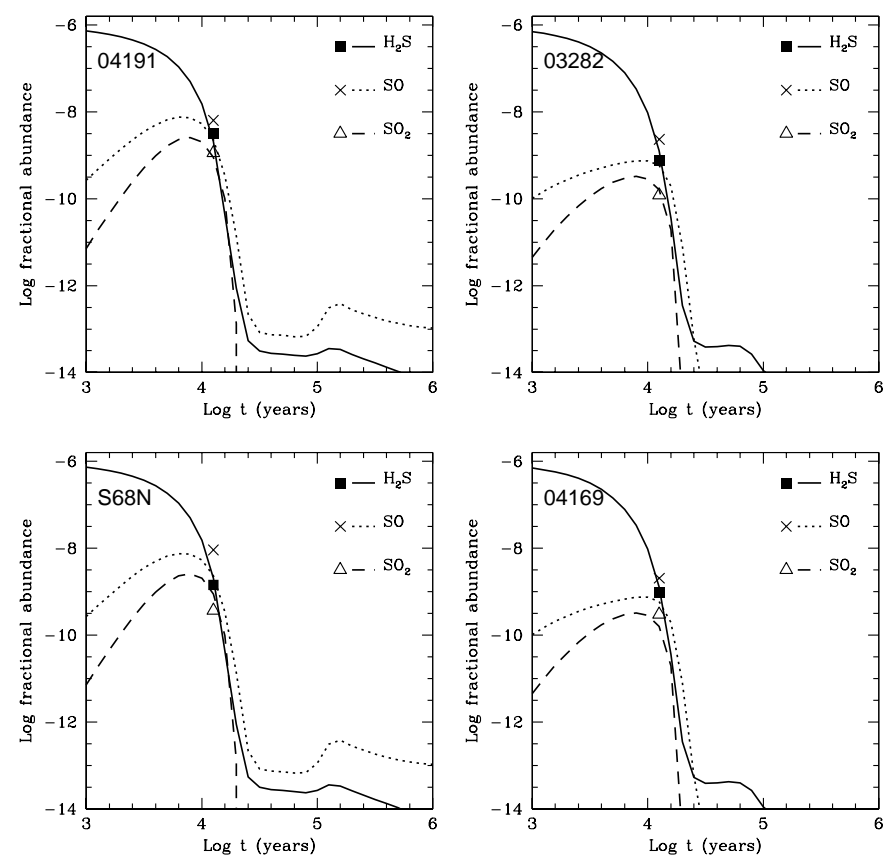

Fig. 11. Plots showing the best match models for the sources where all three sulphur containing molecules were observed. Model parameters are listed in Table 7. Observed abundances are plotted as points.

worse fits. These densities are in the lower range, but not inconsistent with those usually associated with low mass cores and envelopes (van Dishoeck \& Blake 1998).

Temperature does not have a significant affect on the model abundances; these sources with matches at $50 \mathrm{~K}$ kinetic temperatures are nearly as well matched by models with lower kinetic temperatures, when all of the other input parameters remain the same. Similarly, S68N, with a match at a kinetic temperature of $10 \mathrm{~K}$, also has reasonable matches at higher temperatures.

All of these sources are best matched by early timescales, of a few $\times 10^{4}$ years. It may have been expected that IRAS 04169+2702, a Class I source, would have a later chemical evolutionary timescale $\left(\geq 10^{5} \mathrm{yr}\right)$ than the Class 0 sources. However, it is one of the younger Class I sources (Motte \& Andre 2001).

\section{Summary and conclusions}

We have surveyed the $\mathrm{H}_{2} \mathrm{~S}$, SO and $\mathrm{SO}_{2}$ towards 19 Class 0 and Class I sources. The detection of $\mathrm{H}_{2} \mathrm{~S}$ with a fractional abundance around $10^{-9}$ points to the release of this species from the mantles of dust grains in the circumstellar regions of these sources. The abundance of these three sulphur 
containing species has been modelled using a chemical network for the evolution of the sulphur containing species after the injection of $\mathrm{H}_{2} \mathrm{~S}$ released from grain mantles. This model, previously applied to hot cores, is based on the chemical models of Charnley (1997) and Millar et al. (1997). The model can reproduce the simultaneous abundances of $\mathrm{H}_{2} \mathrm{~S}, \mathrm{SO}$ and $\mathrm{SO}_{2}$, at the low temperatures $\left(T_{k} \leq 50 \mathrm{~K}\right)$, and low densities $\left(n\left(\mathrm{H}_{2}\right)<10^{5} \mathrm{~cm}^{-3}\right)$ that are appropriate to low mass dense cores, but the chemistry proceeds more slowly than in hot cores.

Despite the lower temperatures and densities, the models which best fit the observations have a number of similarities to those which fit the observations of hot cores. The models require an initial $\mathrm{H}_{2} \mathrm{~S}$ abundance $>10^{-8}$ in order to reproduce the observed abundances, similar to the values necessary $\left(X\left(\mathrm{H}_{2} \mathrm{~S}\right)=10^{-8}-10^{-6}\right)$ in the hot core model. In addition, like the hot core models, enhanced cosmic ray ionisation rates provide better matches to the observed abundances of all three molecules.

The lower average abundances towards Class I sources as compared to Class 0 sources for $\mathrm{H}_{2} \mathrm{~S}, \mathrm{SO}$ and particularly $\mathrm{SO}_{2}$, suggests that Class I sources are more chemically evolved, with matches to the chemical model suggesting that the Class I sources at least $3 \times 10^{3}$ years older than their Class 0 counterparts, with an upper age limit for the Class I sources (assuming a low-density, low temperature, high $\zeta_{\mathrm{H}}$ sulphur model) of a few $\times 10^{5} \mathrm{yr}$.

If we are underestimating the fractional abundances of $\mathrm{H}_{2} \mathrm{~S}$, for example by under-resolving the regions of $\mathrm{H}_{2} \mathrm{~S}$ emission or assigning them excessive total $\left(\mathrm{H}_{2}\right)$ column densities (Sect. 3.2), then the chemical model is overestimating the chemical timescales. However, higher $\mathrm{H}_{2} \mathrm{~S}$ abundances would also mean that smaller increases in the ionization rate would be necessary to match the data to the canonical Class $0 / I$ timescales using the sulphur model, bringing the ionization rates more in line with the range estimated by some other studies, for example Caselli et al. (1998). On the other hand uncertainty in the SO fractional abundance, and/or overestimating of the $\mathrm{SO}_{2}$ fractional abundances will make relatively smaller differences to the detailed parameters of the particular chemical model which matches the data.

Detailed modelling of a small sample of three Class 0 sources and one Class I source where we detected all of the observed sulphur transitions did not find any evidence of significant differences in the ages between these particular Class 0 and Class I sources. It is possible that differences in the natal environment are veiling any differences between these particular sources, or that there is not sufficient chemical evolution between these four source for our comparison with just three sulphur bearing species to detect.

The time period where the model matches our data is relatively short, and so it should be possible to provide quite accurate ages for the cores, if the physical conditions and molecular excitation were better known. Assigning an accurate age requires a knowledge of the cosmic ray ionisation rate, but even in the absence of this, the model allows comparison of relative ages assuming the ionization rate does not vary significantly from source to source. Also, observations of a wider range

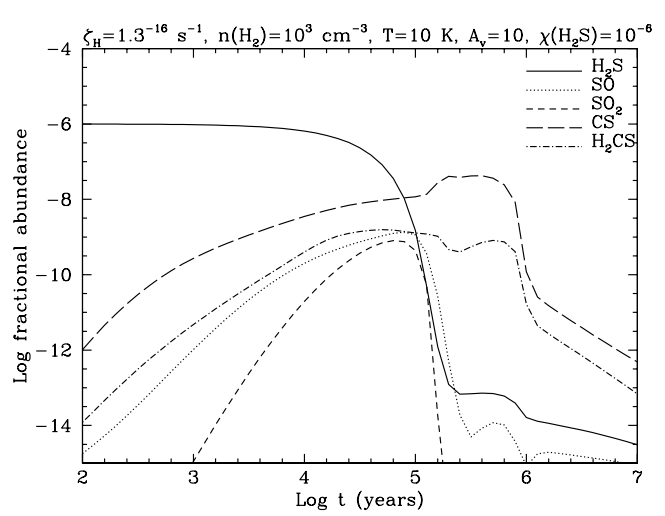

Fig. 12. Example of a chemical model showing the abundances of $C S$ and $\mathrm{H}_{2} \mathrm{CO}$, in addition to $\mathrm{H}_{2} \mathrm{~S}, \mathrm{SO}$ and $\mathrm{SO}_{2}$.

of molecules, to include molecules such as $\mathrm{CS}$, and $\mathrm{H}_{2} \mathrm{CS}$, as shown in Fig. 12, would help constrain the model parameters, and therefore the chemical evolutionary status.

The results presented here show that observations and modelling of sulphur species have the potential to provide a clock measuring the evolution of embedded sources. More observations of sulphur-bearing species in low mass star-forming regions, particularly multi-transition studies at high angular resolution hold the promise of more detailed application of the sulphur chemical clock to arrive at better constrained ages, and in particular relative ages, of embedded sources. Such age estimates should allow a definitive answer to the question of how rapidly Class 0 sources evolve into Class I sources or indeed whether, as some authors have questioned, these sources represent an evolutionary sequence at all (Jayawardhana et al. 2001).

Acknowledgements. Astronomy at UMIST is supported by PPARC. The authors would like to thank Prof. T.J. Millar and Dr. J. Hatchell for advice on aspects of the chemical model.

\section{References}

Bachiller, R., Pérez Gutiérrez, M., Kumar, M. S. N., \& Tafalla, M. 2001, A\&A, 372, 899

Bachiller, R., \& Gutiêrrez, M. P. 1997, ApJ, 487, L93

Bontemps, S., Andre, P., Terebey, S., \& Cabrit, S. 1996, A\&A, 311, 858

Buckle, J. V., \& Fuller, G. A. 2002, A\&A, 381, 77

Buckle, J. V. 2001, in Observational Studies of Low Mass Star Formation, Ph.D. Thesis, Physics Dept., UMIST, Manchester, UK

Caselli, P., Walmsley, C. M., Terzieva, R., \& Herbst, E. 1998, ApJ, 499, 234

Charnley, S. B. 1997, ApJ, 481, 396

Chernin, L., Masson, C. R., \& Fuller, G. A. 1994, ApJ, 436, 741

Davis, C. J., Matthews, H. E., Ray, T. P., et al. 1999, MNRAS, 309, 141

de Boisanger, C., Helmich, F. P., \& van Dishoeck, E. F. 1996, A\&A, 310,315

Dickens, J. E., Irvine, W. M., Snell, R. L., et al. 2000, ApJ, 542, 870

Frerking, M. A., Langer, W. D., \& Wilson, R. W. 1982, ApJ, 262, 590

Fuller, G. A., \& Ladd, E. F. 2002, ApJ, 673, 699

Hatchell, J., Thompson, M. A., Millar, T. J., \& Macdonald, G. H. 1998, A\&A, 338, 713 
Jayawardhana, R., Hartmann, L., \& Calvet, N. 2001, ApJ, 548, 310

Kurtz, S., Cesaroni, R., Churchwell, E., et al. 2000, in Protostars and Planets IV, ed. V. Mannings, A. P. Boss, \& S. S. Russell (University of Arizona Press), 299

Ladd, E. F., Fuller, G. A., \& Deane, J. R. 1998, ApJ, 495, 871

Langer, W. D., van Dishoeck, E. F., Bergin, E. A., et al. 2000, in Protostars and Planets IV, ed. V. Mannings, A. P. Boss, \& S. S. Russell (University of Arizona Press), 29

Millar, T. J., \& Herbst, E. 1990, A\&A, 231, 466

Millar, T. J., Macdonald, G. H., \& Gibb, A. G. 1997, A\&A, 325, 1163

Minh, Y. C., Irvine, W. M., \& Ziurys, L. M. 1989, ApJ, 345, L63

Motte, F., \& Andre, P. 2001, A\&A, 365, 440

Mundy, L. G., Looney, L. W., \& Welch, W. J. 2000, in Protostars and Planets IV, ed. V. Mannings, A. P. Boss, \& S. S. Russell (University of Arizona Press), 335
Pickett, H. M., Poynter, R. L., Cohen, E. A., et al. 1998, in Submillimeter, Millimeter, and Microwave Spectral Line Catalog, J. Quant. Spectrosc. \& Rad. Transfer, 60, 883

Pratap, P., Dickens, J. E., Snell, R. L., et al. 1997, ApJ, 486, 862

Thompson, M. A., Macdonald, G. H., \& Millar, T. J. 1999, A\&A, 342, 809

Tieftrunk, A., Pineau Des Forets, G., Schilke, P., \& Walmsley, C. M. 1994, A\&A, 289, 579

Turner, B. E. 1996, ApJ, 468, 721

van Dishoeck, E. F., \& Black, J. H. 1986, ApJS, 62, 109

van Dishoeck, E. F., \& Blake, G. A. 1998, ARA\&A, 36, 317

van Dishoeck, E. F., \& Hogerheijde, M. 1999, Models and Observations of the Chemistry Near Young Stellar Objects, in The Origin of Stars and Planetary systems, ed. C. J. Lada, \& N. D. Kylafis (Kluwer Academic Publishers), 97 